\title{
Current Operators in Bethe Ansatz and Generalized Hydrodynamics: An Exact Quantum-Classical Correspondence
}

\author{
Márton Borsi, ${ }^{1}$ Balázs Pozsgay, ${ }^{1,2}$ and Levente Pristyák ${ }^{1}$ \\ ${ }^{1}$ Department of Theoretical Physics, Budapest University of Technology and Economics, \\ 1111 Budapest, Budafoki út 8, Hungary \\ ${ }^{2}$ BME Statistical Field Theory Research Group 1111 Budapest, Budafoki út 8, Hungary
}

(Received 9 September 2019; revised manuscript received 9 December 2019; accepted 23 January 2020; published 3 March 2020)

\begin{abstract}
Generalized hydrodynamics is a recent theory that describes large-scale transport properties of onedimensional integrable models. It is built on the (typically infinitely many) local conservation laws present in these systems and leads to a generalized Euler-type hydrodynamic equation. Despite the successes of the theory, one of its cornerstones, namely, a conjectured expression for the currents of the conserved charges in local equilibrium, has not yet been proven for interacting lattice models. Here, we fill this gap and compute an exact result for the mean values of current operators in Bethe ansatz solvable systems valid in arbitrary finite volume. Our exact formula has a simple semiclassical interpretation: The currents can be computed by summing over the charge eigenvalues carried by the individual bare particles, multiplied with an effective velocity describing their propagation in the presence of the other particles. Remarkably, the semiclassical formula remains exact in the interacting quantum theory for any finite number of particles and also in the thermodynamic limit. Our proof is built on a form-factor expansion, and it is applicable to a large class of quantum integrable models.
\end{abstract}

DOI: 10.1103/PhysRevX.10.011054

\section{INTRODUCTION}

The description of the collective motion in many-body quantum systems is one of the most challenging problems in theoretical physics. There are different possible levels for a theoretical treatment, ranging from the microscopic laws to various effective theories describing mesoscopic or macroscopic physics. For large enough systems, one expects that classical behavior will emerge, at least for certain observables. It is thus important to understand how and under what circumstances the various classical theories can be derived from an underlying quantum-mechanical motion [1].

One such classical theory is hydrodynamics: It is known that many quantum systems admit some kind of hydrodynamic description on the mesoscopic and/or macroscopic scales [1,2]. Examples include Bose-Einstein condensates [3] or the quark gluon plasma [4]. Superfluidity is a famous exotic phenomenon, where frictionless flow is realized due to the constraints for the decay of excitations into lower energy modes. Superfluidity has been observed not

Published by the American Physical Society under the terms of the Creative Commons Attribution 4.0 International license. Further distribution of this work must maintain attribution to the author(s) and the published article's title, journal citation, and DOI.
Subject Areas: Quantum Physics, Statistical Physics

only for liquid helium but also in ultracold bosonic and fermionic gases [5].

Another class of systems with exotic hydrodynamic behavior is comprised of the one-dimensional integrable models. In these models, there exist independent conservation laws that constrain the dynamical processes, the number of which grows at least linearly with the volume. As an effect, these models do not thermalize to standard statistical physical ensembles. Instead, the emerging long-time steady states can be described by a generalized Gibbs ensemble (GGE) that involves all higher conserved charges of the model [6,7]. The conservation laws prevent the decay of quasiparticle excitations, and this leads to dissipationless and factorized scattering, which was already demonstrated by experiments [8]. Dissipationless propagation of the collective modes leads to the emergence of ballistic transport and nonzero Drude weights (dc conductivity) [9].

Generalized hydrodynamics (GHD) is a recent theory describing large-scale nonequilibrium behavior in integrable models [10,11] (see also Refs. [12-15]). The theory is built on the local continuity equations following the conservation laws, which lead to a generalized Euler-type equation describing the ballistic transport. The GHD provides exact results for the Drude weights [16,17]. Diffusive corrections to the ballistic transport were also considered in Refs. [18,19], including an exact computation of the diffusion coefficients. Remarkably, the 
predictions of the GHD have already been confirmed in a concrete experimental setup [20].

Despite the successes of the GHD, one of the cornerstones of the theory has not yet been proven. The works $[10,11]$ conjectured an expression for the expectation values of the current operators in local equilibrium, which is central to the derivation of the main equations of motion. Regarding integrable quantum field theories, a proof was provided in Refs. [10,21], whereas for the spin current of the $X X Z$ model, it was proven in Ref. [22]. Nevertheless, for arbitrary current operators in interacting lattice models or nonrelativistic gases (the models most relevant to experiments), it was completely missing up to now. It is the goal of this paper to provide a proof of the conjecture valid for a wide class of Bethe ansatz solvable models.

The problem of the current mean values is also interesting from a purely theoretical perspective, without the immediate application to GHD. A large body of literature has already addressed equilibrium correlation functions in Bethe ansatz solvable models [23-32], with particular interest devoted to the asymptotics of two-point functions (for a review, see Ref. [33]) and to equilibrium mean values of arbitrary short-range operators of the Heisenberg spin chains (for a review, see Ref. [34]). In contrast, the current operators are very specific short-range objects, and as we show, their finite-volume mean values take a remarkably simple form. This result has not yet been noticed in the Bethe ansatz literature, and we believe that it deserves a study in its own right.

In the following subsection, we describe the conjecture of Refs. $[10,11]$ for the current mean values and explain its role in the GHD, while omitting many technical details. This brief introduction motivates our finite-volume investigations. After that, the remainder of this paper is composed as follows: In Sec. II, we specify the problem and present our main new results, with a semiclassical interpretation given in Sec. III. Section IV includes our modelindependent proof based on a finite-volume form-factor expansion. Section V includes known generalities about the charge and current operators in integrable lattice models; also, it gives a short summary of the algebraic Bethe ansatz. The proof of our form-factor expansion for the $X X Z$ and $X X X$ spin chains is presented in Sec. VI. In Sec. VII, we point out a connection to the theory of factorized correlation functions. Finally, we conclude in Sec. VIII.

\section{A. Foundations of the GHD}

Isolated integrable models equilibrate to steady states described by generalized GGEs. Each GGE can be characterized by a set of parameters (generalized temperatures), or alternatively, by the mean values of all local and quasilocal conserved charges in the model $[7,35,36]$.

The timescales of equilibration to the GGE are set by the microscopic laws. It follows that in mesoscopic or macroscopic dynamical processes, local equilibration happens much sooner than the characteristic times of the transport processes. This separation of timescales leads to the hydrodynamic description: GHD assumes the existence of fluid cells (regions in space much larger than the interparticle distance and much smaller than the variation of the physical observables) such that the state of each fluid cell can be described by a local GGE. The parameters of these local GGEs are then space and time dependent.

The generalized eigenstate thermalization hypothesis (GETH) [37] states that in local equilibrium the local observables depend only on the mean values of the conserved charges and not on any other particular detail of the states. Thus, in order to describe the dynamical processes, it is enough to establish flow equations for the conserved charges, which will then determine all other physical observables on the hydrodynamic scales.

For simplicity, let us consider here a continuum model in the thermodynamic limit. Let the complete set of local and/or quasilocal conserved charges be $Q_{\alpha}=\int_{-\infty}^{\infty} d x Q_{\alpha}(x)$, with $\alpha$ being an index or a multi-index, and $Q_{\alpha}(x)$ being the charge-density operators. Conservation of the charges implies that there exist current operators $J_{\alpha}(x)$ satisfying the equation of motion in the Heisenberg picture:

$$
\partial_{t} Q_{\alpha}(x, t)+\partial_{x} J_{\alpha}(x, t)=0 .
$$

GHD concerns the mean values of these relations:

$$
\partial_{t}\left\langle Q_{\alpha}(x, t)\right\rangle+\partial_{x}\left\langle J_{\alpha}(x, t)\right\rangle=0 .
$$

A closed set of flow equations can be obtained if the currents are expressed using only local information about the charges. In hydrodynamics, this task is performed through a derivative expansion:

$$
\left\langle J_{\alpha}(x, t)\right\rangle=f_{\alpha}\left[\left\{Q_{\beta}(x, t), \partial_{x} Q_{\beta}(x, t), \ldots\right\}_{\beta=1,2, \ldots}\right] .
$$

In the first approximation, we neglect the spatial variations and express the currents in the fluid cells using the mean values $\left\langle Q_{\beta}(x, t)\right\rangle$ in that specific fluid cell only. This approximation describes the ballistic part of the transport. The diffusive part of the transport can also be treated by considering the derivatives $\partial_{x}\left\langle Q_{\beta}(x, t)\right\rangle$ [19], but this is not considered here.

The ballistic flow equations then follow from Eq. (1.2), given that one can compute the exact mean values of the currents in the local equilibrium states as a function of the charges or using any alternative description of the local GGEs. We consider this problem in the present paper.

A large class of integrable models is solvable by the Bethe ansatz [38]; prominent examples include the Heisenberg spin chains or the 1D Bose gas with pointlike interaction. In these models, the local equilibria in the thermodynamic limit can be characterized by the root densities $\varrho_{n}(\lambda)$ of the interacting quasiparticles, where $n$ 
stands for a particle type, and $\lambda$ is the so-called rapidity parameter. The root densities can be understood as generalizations of momentum-dependent occupation numbers in free theory. Dissipationless scattering implies that these densities are well-defined concepts even in the presence of interactions. The construction of the GGE is equivalent to specifying all the root densities $Q_{n}(\lambda)$ because they carry all the information about the local equilibria. This equivalence is the ultimate form of the GETH, and it was understood in Refs. [36,39] following the earlier works [40-45].

In GHD, we thus need to specify the Bethe root densities for each fluid cell; therefore, they will depend also on the $x, t$ coordinates of the cell. It is a very fruitful idea of the GHD that instead of concentrating on Eq. (1.2) for the charges, one should derive flow equations for the rapidity distribution functions. This can be achieved starting from Eq. (1.2) by expressing both mean values in the continuity equations using the densities $\varrho_{n}(\lambda)$ only.

The mean values of the charges can be computed additively. In local equilibrium, we have

$$
\left\langle Q_{\alpha}\right\rangle=\int d \lambda \varrho(\lambda) q_{\alpha}(\lambda),
$$

where $q_{\alpha}(\lambda)$ is the single-particle eigenvalue of the charges, and here we assume only one particle species for simplicity. In GHD, Eq. (1.4) is assumed to hold for each fluid cell separately.

For the currents, it was conjectured in Refs. [10,11] that the mean values can be computed using a semiclassical expression, namely, by integrating over the carried charge multiplied by an effective propagation speed:

$$
\left\langle J_{\alpha}\right\rangle=\int d \lambda \varrho(\lambda) v_{\mathrm{eff}}(\lambda) q_{\alpha}(\lambda) .
$$

Here, the effective speed $v_{\text {eff }}(\lambda)$ is a generalization of the one-particle group velocity, which also takes into account the interactions between the particles. It has a physical explanation using a semiclassical argument: The oneparticle wave packets suffer time delays due to the scattering on the other particles, and these time delays accumulate along the orbit, eventually modifying the propagation speed. The resulting effective speed $v_{\text {eff }}(\lambda)$ is a collective property of the local GGE because for each $\lambda$ it also depends on the particle density $\varrho\left(\lambda^{\prime}\right)$ for all other $\lambda^{\prime}$. For a precise definition of $v_{\text {eff }}(\lambda)$, we refer to Refs. [10,11].

Equation (1.5) has not yet been proven in the Bethe ansatz. It is our goal to fill this gap and to prove Eq. (1.5) starting from a rigorous finite-volume computation.

Regarding the interpretation of Eq. (1.5), it was explained in Ref. [46] that the GHD can be simulated by the so-called "flea gas" model, which describes 1D motion of purely classical particles subject to time delays (displacements) as an effect of interparticle collisions. Thus, one observes a complete quantum-classical correspondence on the hydrodynamic scale.

In this work, we show that the functional form of the mean values is the same in finite and infinite volume; therefore, the quantum-classical correspondence holds with an arbitrary finite number of particles.

\section{CURRENT MEAN VALUES}

\section{A. Elements of integrability}

We consider integrable many-body quantum models, including both lattice and continuum theories [23]. In this work, we limit ourselves to those theories where particle number is conserved and which can be solved by the traditional ("non-nested") Bethe ansatz. The main examples are given by the Heisenberg spin chains, the 1D Bose gas, and also certain integrable QFTs [23]. Regarding other types of integrable models, we give a few comments in the Conclusions.

In this section, we present formulas pertaining to lattice models. We consider an integrable Hamiltonian $H$ in a finite-volume $L$ :

$$
H=\sum_{x=1}^{L} h(x) .
$$

Here, $h(x)$ is the Hamiltonian density. In the most relevant cases, $h(x)$ is a two-site operator, but we do not necessarily need this restriction. For simplicity, we require periodic boundary conditions.

In integrable models, there exists a family of conserved operators $Q_{\alpha}$, where $\alpha$ is an index or multi-index. They mutually commute,

$$
\left[Q_{\alpha}, Q_{\beta}\right]=0,
$$

and the Hamiltonian is a member of the series.

We concentrate on the strictly local operators, which are given as

$$
Q_{\alpha}=\sum_{x=1}^{L} Q_{\alpha}(x),
$$

where $Q_{\alpha}(x)$ is a short-range operator identified as the charge density. It is important that in a finite volume, $Q_{\alpha}$ is well defined for volumes larger than the range of $Q_{\alpha}(x)$, and then the density $Q_{\alpha}(x)$ does not depend further on $L$. Details on the canonical construction of the charges and a few concrete examples are given in Sec. V.

The Hamiltonian is a member of the series; thus, the global operator $Q_{\alpha}$ is conserved:

$$
\frac{d}{d t} Q_{\alpha}=i\left[H, Q_{\alpha}\right]=0 .
$$


We are interested in nonequilibrium processes and a hydrodynamic description; therefore, we investigate the time evolution of the charge contained in a finite section. This problem leads to a continuity relation in operator form:

$\frac{d}{d t} \sum_{x=x_{1}}^{x_{2}} Q_{\alpha}(x)=i\left[H, \sum_{x=x_{1}}^{x_{2}} Q_{\alpha}(x)\right]=J_{\alpha}\left(x_{1}\right)-J_{\alpha}\left(x_{2}+1\right)$.

This relation defines the current operator $J_{\alpha}(x)$ associated with $Q_{\alpha}(x)$ under the time evolution of $H$. The existence of $J_{\alpha}(x)$ follows simply from Eq. (2.4) and locality arguments.

The relations (2.3) and (2.5) do not define the $Q_{\alpha}(x)$ and $J_{\alpha}(x)$ operators uniquely; certain subtleties are discussed in Sec. V. We just put forward that the additive normalization of the current operators is chosen by the physical requirement that

$$
\left\langle 0\left|J_{\alpha}(x)\right| 0\right\rangle=0,
$$

where $|0\rangle$ is the vacuum or reference state with no particles.

Our goal is to determine the mean values

$$
\left\langle n\left|J_{\alpha}(x)\right| n\right\rangle,
$$

where $|n\rangle$ is an arbitrary excited state of the finite-volume Hamiltonian. In the thermodynamic limit, these mean values will enter the flow equations (1.2).

In the models in question, the exact eigenstates are found using the Bethe ansatz $[23,38]$. The states are characterized by a set of lattice momenta $\left\{p_{1}, \ldots, p_{N}\right\}$ that describes the interacting spin waves.

The unnormalized Bethe wave function can be written for $x_{1}<x_{2}<\ldots<x_{N}$ as [38]

$\Psi\left(x_{1}, x_{2}, \ldots, x_{N}\right)=\sum_{\sigma \in S_{N}}\left[\exp \left(i \sum_{j=1}^{N} p_{\sigma_{j}} x_{j}\right) \prod_{\substack{j<k \\ \sigma_{j}>\sigma_{k}}} S\left(p_{j}, p_{k}\right)\right]$.

Here, each term in the sum represents free wave propagation with a given spatial ordering of the particles. The amplitude $S\left(p_{j}, p_{k}\right)=e^{i \delta\left(p_{j}, p_{k}\right)}$ is a relative phase between terms with different particle ordering, and it can be interpreted as the two-particle scattering amplitude. It depends on the model in question and can be determined from the two-particle problem. The wave function is twoparticle reducible: Any multiparticle interaction is explicitly factorized into a succession of two-particle scatterings.

These wave functions describe eigenstates if they are periodic, from which we obtain the Bethe equations:

$$
e^{i p_{j} L} \prod_{k \neq j} S\left(p_{j}, p_{k}\right)=1, \quad j=1, \ldots, N .
$$

It is useful to introduce the rapidity parametrization $p=p(\lambda)$, where $\lambda$ is the additive parameter for the scattering phase:

$$
S\left(p_{j}, p_{k}\right)=S\left[p\left(\lambda_{j}\right), p\left(\lambda_{k}\right)\right]=S\left(\lambda_{j}-\lambda_{k}\right) .
$$

The Bethe equations can then be written as

$$
e^{i p\left(\lambda_{j}\right) L} \prod_{k \neq j} S\left(\lambda_{j}-\lambda_{k}\right)=1, \quad j=1, \ldots, N .
$$

In the following, the normalized $N$-particle Bethe states with rapidities $\{\lambda\}_{N}=\left\{\lambda_{1}, \ldots, \lambda_{N}\right\}$ are denoted as $\left|\{\lambda\}_{N}\right\rangle$.

The total energy and lattice momentum can be computed additively:

$$
\begin{aligned}
& E=\sum_{j=1}^{N} e\left(\lambda_{j}\right), \\
& P=\sum_{j=1}^{N} p\left(\lambda_{j}\right) \quad \bmod 2 \pi,
\end{aligned}
$$

where the single-particle energy $e(\lambda)$ is a further characteristic function of the model.

Similarly, the eigenvalues of the conserved charges are

$$
Q_{\alpha}\left|\lambda_{1}, \ldots, \lambda_{N}\right\rangle=\left[\sum_{j=1}^{N} q_{\alpha}\left(\lambda_{j}\right)\right]\left|\lambda_{1}, \ldots, \lambda_{N}\right\rangle,
$$

where $q_{\alpha}(\lambda)$ are the one-particle eigenvalues.

For later use, let us write the Bethe equations in the logarithmic form:

$p\left(\lambda_{j}\right) L+\sum_{k \neq j} \delta\left(\lambda_{j}-\lambda_{k}\right)=2 \pi I_{j}, \quad j=1, \ldots, N$.

Here, $I_{j} \in \mathbb{Z}$ are the momentum quantum numbers, which can be used to parametrize the states.

In our derivation, an important role is played by the so-called Gaudin matrix $G$, which is defined as

$$
G_{j k}=\frac{\partial}{\partial \lambda_{k}}\left(2 \pi I_{j}\right), \quad j, k=1, \ldots, N,
$$

where now the $I_{j}$ are regarded as functions of the rapidities. Explicitly, we have

$G_{j k}=\delta_{j k}\left[p^{\prime}\left(\lambda_{j}\right) L+\sum_{l=1}^{N} \varphi\left(\lambda_{j}-\lambda_{l}\right)\right]-\varphi\left(\lambda_{j}-\lambda_{k}\right)$,

where

$$
\varphi(\lambda)=-i \frac{\partial}{\partial \lambda} \log [S(\lambda)]
$$


There are two interpretations of the Gaudin matrix. First, $\operatorname{det} G$ describes the density of states in rapidity space. This follows from the fact that in the space of the quantum numbers, the states are evenly distributed, and $G$ is defined as the Jacobian of the mapping from $\lambda_{j}$ to $I_{j}$. Second, the Gaudin determinant also describes the norm of the Bethe ansatz wave function in many integrable models (see Sec. V and Refs. [47,48]).

\section{B. Main result}

Our main result for the normalized mean values of the current operators is the following:

$$
\left\langle\{\lambda\}_{N}\left|J_{\alpha}(x)\right|\{\lambda\}_{N}\right\rangle=\mathbf{e}^{\prime} \cdot G^{-1} \cdot \mathbf{q}_{\alpha} .
$$

Here, the quantities $\mathbf{e}^{\prime}$ and $\mathbf{q}_{\alpha}$ are $N$-dimensional vectors with elements

$$
\left(\mathbf{e}^{\prime}\right)_{j}=\frac{\partial e\left(\lambda_{j}\right)}{\partial \lambda}, \quad\left(\mathbf{q}_{\alpha}\right)_{j}=q_{\alpha}\left(\lambda_{j}\right),
$$

and $G^{-1}$ is the inverse of the Gaudin matrix.

In the simplest case of $N=1$, the Gaudin matrix has a single element $G_{11}=L p^{\prime}(\lambda)$, and Eq. (2.18) gives the anticipated classical result

$$
\left\langle\lambda\left|J_{\alpha}(x)\right| \lambda\right\rangle=\frac{e^{\prime}(\lambda) q_{\alpha}(\lambda)}{L p^{\prime}(\lambda)}=\frac{v(\lambda) q_{\alpha}(\lambda)}{L},
$$

where we introduce the bare group velocity $v(\lambda)=$ $e^{\prime}(\lambda) / p^{\prime}(\lambda)=\partial e / \partial p$.

A similar semiclassical interpretation can be given also for higher particle numbers. Using the definition (2.15) and the additive formula (2.12), we find the alternative expression

$$
\left\langle\{\lambda\}_{N}|J(x)|\{\lambda\}_{N}\right\rangle=\frac{1}{L} \sum_{j=1}^{N} v_{\text {eff }}\left(\lambda_{j}\right) q_{\alpha}\left(\lambda_{j}\right),
$$

where we define the quantities

$$
v_{\text {eff }}\left(\lambda_{j}\right)=\frac{L}{2 \pi} \frac{\partial E}{\partial I_{j}} .
$$

In Sec. III, it is explained that the $v_{\text {eff }}$ can be understood in a simple semiclassical picture as effective velocities describing the propagation of the individual bare particles in the presence of the others.

It is remarkable that the exact result and the functional form of the effective velocity are so simple in the finitevolume situation. In the thermodynamic limit, the papers $[10,11]$ conjectured the formula (1.5) with the effective speed given by

$$
v_{\text {eff }}(\lambda)=\frac{\partial \varepsilon(\lambda)}{\partial P(\lambda)},
$$

where $\varepsilon(\lambda)$ and $P(\lambda)$ are the so-called "dressed energy" and "dressed momentum." These are computed as the energy and momentum differences as we add a particle with rapidity $\lambda$ into a sea of particles. The "dressing" takes into account the backflow of the other particles, which can be computed from the Bethe equations (2.14).

The correspondence between Eq. (2.23) and our Eq. (2.22) is evident: Small changes in the dressed momentum and dressed energy can be traced back to small changes in the momentum quantum numbers and the overall finite-volume energy, respectively,

$$
\delta \varepsilon\left(\lambda_{j}\right) \sim \delta E, \quad \delta P\left(\lambda_{j}\right) \sim \delta\left(\frac{2 \pi I_{j}}{L}\right) .
$$

This correspondence implies that Eq. (2.23) is indeed the thermodynamic limit of our Eq. (2.22). Furthermore, our Eq. (2.21) can be seen as the finite-volume origin of the thermodynamic formula (1.5).

Equation (2.18) is exact in those cases when the Bethe wave function is exact; this holds for integrable spin chains or the 1D Bose gas. On the other hand, in integrable QFT (IQFT) in finite volume, the Bethe wave function is only an approximation, and in IQFT, Eq. (2.18) holds up to exponentially small corrections in the volume.

Depending on the model, the bare particles of the Bethe ansatz can form bound states. These bound states are described by the so-called string solutions of the Bethe equations [49]. It is important that our formula (2.18) is exact on the level of the individual Bethe rapidities, even in the presence of strings. An effective description involving the string centers (describing the rapidities of the composite particles) can be given afterward using well-established methods [50].

The main result (2.18) concerns the physical current operators that describe the flow under time evolution by the physical Hamiltonian. However, it is also useful to consider certain generalized current operators that describe the flow of a given charge under time evolution generated by some other charge; such operators were already studied in Ref. [12].

We thus consider two local charges $Q_{\alpha}$ and $Q_{\beta}$ belonging to the same integrable hierarchy, implying that all three operators $H, Q_{\alpha}, Q_{\beta}$ commute with each other. We define $J_{\alpha}^{\beta}$ to be the current of the charge $Q_{\alpha}$ under unitary time evolution dictated by $Q_{\beta}$ :

$$
i\left[Q_{\beta}, \sum_{x=x_{1}}^{x_{2}} Q_{\alpha}(x)\right]=J_{\alpha}^{\beta}\left(x_{1}\right)-J_{\alpha}^{\beta}\left(x_{2}+1\right) .
$$

Locality of the charge densities $Q_{\alpha, \beta}(x)$ and the global relation $\left[Q_{\alpha}, Q_{\beta}\right]=0$ implies that the operator 
equation (2.25) can always be solved with some shortrange $J_{\alpha}^{\beta}(x)$.

For the mean values of these generalized current operators, we have the following result:

$$
\left\langle\{\lambda\}_{N}\left|J_{\alpha}^{\beta}(x)\right|\{\lambda\}_{N}\right\rangle=\mathbf{q}_{\beta}^{\prime} \cdot G^{-1} \cdot \mathbf{q}_{\alpha} .
$$

Here, $\mathbf{q}_{\beta}^{\prime}$ is an $N$-element vector with components $q_{\beta}^{\prime}\left(\lambda_{j}\right)$, where $q_{\beta}(\lambda)$ is the one-particle eigenvalue of the charge $Q_{\beta}$ and the prime denotes differentiation. The analogy between Eqs. (2.26) and (2.18) is evident: Only the one-particle eigenvalues of the time-evolution operator are replaced. A special case and certain symmetry properties of this general statement are treated in the Appendixes A and B.

In the following section, we describe a semiclassical interpretation of these results, whereas the full quantummechanical proof is provided in Sec. IV.

\section{THE SEMICLASSICAL INTERPRETATION}

Here we present a semiclassical computation, which also gives a simple physical interpretation for the main result (2.18). Our arguments are very similar to those presented in Ref. [46], with the main difference being that we consider finite systems: We are looking at the motion of $N$ particles on a finite ring of volume $L$ (see Fig. 1). For convenience, we consider here continuum models and a strictly pointlike interaction.

Instead of solving the time-dependent Schrödinger equation, we employ a semiclassical picture; namely, we assume that the particles can be assigned well-defined straight orbits as long as they do not interact with each other. In this picture, a particle with rapidity $\lambda$ is represented by a wave packet, which travels with the group velocity

$$
v(\lambda)=\frac{d e}{d p}=\frac{e^{\prime}(\lambda)}{p^{\prime}(\lambda)}
$$

In a typical situation, all speeds are different and particles meet as they travel around the volume. The scattering events are taken into account by using an exact quantummechanical solution of the two-body problem. For pure Fourier modes, this method implies that for the scattering of particle $j$ on $k$ the wave function has to be multiplied by the

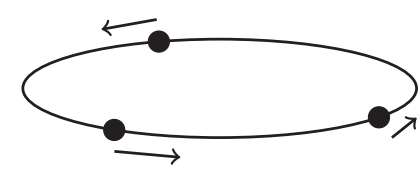

FIG. 1. The semiclassical picture for the effective velocities. There are $N$ particles moving on a circle of circumference $L$. The only effect of the interaction is the time delays after the scattering events. These delays accumulate and lead to well-defined effective speeds in the long time limit. phase $S\left(\lambda_{j}-\lambda_{k}\right)$; this phase factor is also reflected by the Bethe ansatz wave function (2.8). This momentum-dependent phase results in displacements of the center of the wave packets [51-53]. Such time delays are also present in classical integrable models, including models supporting solitons [54] or the hard rod gas [55].

After a displacement process, the particles continue their path with their own bare speeds until a further scattering event occurs. The time delays suffered in each of these events accumulate, and this alters the actual propagation speed of the wave packets, leading to the emergence of effective velocities $v_{\text {eff }}\left(\lambda_{j}\right)$. It is important that due to the higher conservation laws, the multiparticle scattering events always factorize into a succession of two-particle scatterings [56], which also implies that the particle rapidities never change during time evolution.

In this semiclassical picture, the current mean values are evaluated simply as

$$
J_{\alpha, c l}=\frac{1}{L} \sum_{j=1}^{N} v_{\mathrm{eff}}\left(\lambda_{j}\right) q_{\alpha}\left(\lambda_{j}\right) .
$$

Our goal is to find the emerging effective velocities.

We consider long times such that each pair of particles has scattered on each other many times. In this limit, the particular order of the individual scattering events (which depends on the initial positions of the particles) becomes irrelevant.

The spatial displacement of particle $j$ caused by the scattering on the particle $k(j<k)$ is given by the derivative [51-53]

$$
\Delta s_{j k}=\frac{\partial \delta\left(p_{j}, p_{k}\right)}{\partial p_{j}}=\frac{\varphi\left(\lambda_{j}-\lambda_{k}\right)}{p^{\prime}\left(\lambda_{j}\right)},
$$

where we use the rapidity parametrization, and $\varphi(u)$ is defined in Eq. (2.17). This formula is valid when particle $j$ overtakes particle $k$ from the left, i.e., when $v_{j}>v_{k}$. We can formally extend it as

$$
\Delta s_{j k}=\sigma_{j k} \frac{\varphi\left(\lambda_{j}-\lambda_{k}\right)}{p^{\prime}\left(\lambda_{j}\right)},
$$

where

$$
\sigma_{j k}= \begin{cases}+1 & \text { if } v_{\text {eff }}\left(\lambda_{j}\right)>v_{\text {eff }}\left(\lambda_{k}\right), \\ -1 & \text { if } v_{\text {eff }}\left(\lambda_{j}\right)<v_{\text {eff }}\left(\lambda_{k}\right) .\end{cases}
$$

The time elapsed between the two scattering events of the same two particles $j$ and $k$ can be expressed as

$$
T_{j k}=\frac{L}{\left|v_{\text {eff }}\left(\lambda_{j}\right)-v_{\text {eff }}\left(\lambda_{k}\right)\right|} .
$$


For asymptotically long times $t$, the accumulated displacement that particle $j$ suffers is given by

$$
\Delta s_{j}(t)=\sum_{\substack{k=1 \\ k \neq j}}^{N} \Delta s_{j k} \cdot \frac{t}{T_{j k}}
$$

This displacement causes the difference between the effective and bare velocities:

$$
\Delta s_{j}(t)=\left(v\left(\lambda_{j}\right)-v_{\mathrm{eff}}\left(\lambda_{j}\right)\right) t
$$

Putting everything together, we obtain the self-consistent relation

$v\left(\lambda_{j}\right)=v_{\text {eff }}\left(\lambda_{j}\right)+\frac{1}{L} \sum_{\substack{k=1 \\ k \neq j}}^{N} \Delta s_{j k}\left|v_{\text {eff }}\left(\lambda_{j}\right)-v_{\text {eff }}\left(\lambda_{k}\right)\right|$.

As an effect of the extra sign $\sigma_{j k}$ in Eq. (3.4), it can be written as

$v\left(\lambda_{j}\right)=v_{\text {eff }}\left(\lambda_{j}\right)+\frac{1}{L} \sum_{\substack{k=1 \\ k \neq j}}^{N} \frac{\varphi_{j k}}{p^{\prime}\left(\lambda_{j}\right)}\left[v_{\text {eff }}\left(\lambda_{j}\right)-v_{\text {eff }}\left(\lambda_{k}\right)\right]$.

Multiplying by $L p^{\prime}\left(\lambda_{j}\right)$ and using the definition of the bare group velocity, we get

$e^{\prime}\left(\lambda_{j}\right) L=L p^{\prime}\left(\lambda_{j}\right) v_{\text {eff }}\left(\lambda_{j}\right)+\sum_{\substack{k=1 \\ k \neq j}}^{N} \varphi_{j k}\left[v_{\text {eff }}\left(\lambda_{j}\right)-v_{\text {eff }}\left(\lambda_{k}\right)\right]$.

On the rhs, we can recognize the action of the Gaudin matrix (2.16), and we can thus write

$$
L \mathbf{e}^{\prime}=G \cdot \mathbf{v}_{\text {eff }} .
$$

Multiplying by $G^{-1}$ and substituting the $v_{\text {eff }}\left(\lambda_{j}\right)$ into Eq. (3.2) leads to

$$
J_{\alpha, c l}=\mathbf{e}^{\prime} \cdot G^{-1} \cdot \mathbf{q}_{\alpha},
$$

which is identical to the full quantum-mechanical result (2.18). We thus demonstrate a complete quantum-classical correspondence with a finite number of particles.

In our derivation, we assume that $v_{\text {eff }}\left(\lambda_{j}\right)>v_{\text {eff }}\left(\lambda_{k}\right)$ whenever $v\left(\lambda_{j}\right)>v\left(\lambda_{k}\right)$. However, as natural as this requirement may seem, there can be situations where it does not hold [57]. In those cases, the quantum result remains valid, but the semiclassical picture cannot be applied.

\section{PROOF USING A FORM-FACTOR EXPANSION}

Here we present a model-independent proof of our main result for the current mean values. Our technique relies on a finite-volume form-factor expansion theorem. The proof of this expansion theorem can depend on the particular model, but the computations of this section are quite general.

For the most part, we concentrate only on the physical currents $J_{\alpha}$. The generalized currents defined in Eq. (2.25) are considered in Sec. IV C.

The starting point is to use the definition of the current operators (2.5) and to consider matrix elements of this operator relation. We intend to compute the mean values of the currents, but taking the mean values of Eq. (2.5) automatically gives zero on both sides due to the Bethe states being translationally invariant and eigenstates of $H$. Instead, it is useful to take the off-diagonal matrix elements between two Bethe states with a nonequal total lattice momentum:

$$
\begin{aligned}
& i\left(\sum_{j=1}^{N} e\left(\lambda_{j}\right)-e\left(\mu_{j}\right)\right)\left\langle\{\lambda\}_{N}\left|Q_{\alpha}(x)\right|\{\mu\}_{N}\right\rangle \\
& \quad=\left(1-\prod_{j=1}^{N} e^{i\left[p\left(\mu_{j}\right)-p\left(\lambda_{j}\right)\right]}\right)\left\langle\{\lambda\}_{N}\left|J_{\alpha}(x)\right|\{\mu\}_{N}\right\rangle .
\end{aligned}
$$

In integrable models, generic off-diagonal finite-volume matrix elements of local operators can be expressed as

$$
\left\langle\{\lambda\}_{N}|\mathcal{O}(0)|\{\mu\}_{M}\right\rangle=\frac{F^{\mathcal{O}}\left(\{\lambda\}_{N} \mid\{\mu\}_{M}\right)}{\operatorname{det} G_{\mu} \operatorname{det} G_{\lambda}}
$$

where the function $F^{\mathcal{O}}\left(\{\lambda\}_{N} \mid\{\mu\}_{M}\right)$ is the so-called form factor, and $\operatorname{det} G_{\lambda}$ and $\operatorname{det} G_{\mu}$ describe the norm of the Bethe ansatz wave function, or alternatively, the density of Bethe states in rapidity space. They are $N \times N$ and $M \times M$ Gaudin determinants computed from the sets of rapidities $\{\lambda\}_{N}$ and $\{\mu\}_{M}$.

The form factors describe the transition-matrix element for the unnormalized Bethe wave functions (7.5). They are meromorphic functions, and they are completely independent from the volume. The volume dependence of the physical matrix elements comes only through the solution of the Bethe equations and the normalization factors. The properties of the form factors have been investigated both in QFT [58] and using the algebraic Bethe ansatz (ABA) [23]. A derivation of the analytic properties in the Lieb-Liniger model was also given using the coordinate Bethe ansatz in Ref. [59]. The statement [60] is well known in the literature dealing with integrable lattice models [23], and for integrable QFT, it was first written down in Ref. [60].

As opposed to the transition-matrix elements, the mean values of local operators in Bethe states cannot be expressed directly using the infinite-volume form factors. 
The reason is the appearance of the so-called disconnected terms: On a mathematical level, they arise from the kinematical poles of the form factors, whereas their physical interpretation is that they describe processes when a subset of the particles does not interact with the local operator. On the other hand, those processes when some of the particles do interact with the operator are described by certain diagonal limits of the form-factor functions.

In order to describe the finite-volume mean values, let us define the so-called symmetric evaluation of the diagonal form factors as

$$
F_{s}^{\mathcal{O}}\left(\{\lambda\}_{N}\right)=\lim _{\varepsilon \rightarrow 0} F^{\mathcal{O}}\left(\lambda_{1}+\varepsilon, \ldots, \lambda_{N}+\varepsilon \mid \lambda_{N}, \ldots, \lambda_{1}\right) .
$$

There is another often used diagonal limit called the connected form factors, but they are not used in this work, and for a thorough discussion, we refer to Ref. [61].

It is useful to define the functions $\rho_{N}\left(\lambda_{1}, \ldots, \lambda_{N}\right)$ as the $N \times N$ Gaudin determinants evaluated at the set of rapidities $\left\{\lambda_{1}, \ldots, \lambda_{N}\right\}$. In the notations, we suppress the index $N$ and write simply

$$
\rho\left(\{\lambda\}_{N}\right)=\operatorname{det} G_{\lambda} .
$$

We remind that the Gaudin determinants describe the norms of Bethe wave functions for eigenstates, i.e., for sets of rapidities satisfying the Bethe equations. On the other hand, the functions $\rho\left(\{\lambda\}_{N}\right)$ are defined for arbitrary sets of rapidities.

It is useful to write down the first two $\rho\left(\{\lambda\}_{N}\right)$ functions. For $N=1$, we have simply

$$
\rho(\lambda)=L p^{\prime}(\lambda)
$$

For $N=2$, the Gaudin matrix is

$$
G=\left(\begin{array}{cc}
p^{\prime}\left(\lambda_{1}\right) L+\varphi_{12} & -\varphi_{12} \\
-\varphi_{12} & p^{\prime}\left(\lambda_{2}\right) L+\varphi_{12}
\end{array}\right),
$$

and its determinant is

$\rho\left(\lambda_{1}, \lambda_{2}\right)=L^{2} p^{\prime}\left(\lambda_{1}\right) p^{\prime}\left(\lambda_{2}\right)+L\left[p^{\prime}\left(\lambda_{1}\right)+p^{\prime}\left(\lambda_{2}\right)\right] \varphi_{12}$,

where $\varphi_{12}=\varphi\left(\lambda_{1}-\lambda_{2}\right)$.

Our proof for the current mean values is based on the following expansion theorem.

Theorem 1 The finite-volume mean values of local operators can be computed through the expansion

$\left\langle\{\lambda\}_{N}|\mathcal{O}(0)|\{\lambda\}_{N}\right\rangle=\frac{\sum_{\left\{\lambda^{+}\right\} \cup\left\{\lambda^{-}\right\}} F_{S}^{\mathcal{O}}\left(\left\{\lambda^{+}\right\}\right) \rho\left(\left\{\lambda^{-}\right\}\right)}{\rho(\{\lambda\})}$,

where the summation runs over all partitionings of the set of the rapidities into $\left\{\lambda^{+}\right\} \cup\left\{\lambda^{-}\right\}$. The partitionings include those cases where either subset is the empty set, and in these cases, it is understood that $\rho(\varnothing)=1$, and $F_{s}^{\mathcal{O}}(\varnothing)=\langle\mathcal{O}\rangle$ is the VEV. The relation [61] is exact when the Bethe ansatz wave functions are exact eigenstates of the model.

This theorem was first formulated in Ref. [61] for integrable QFT, where the Bethe ansatz for the finitevolume eigenstates is not exact due to the presence of virtual particles. Therefore, in IQFT the theorem holds up to corrections exponentially small in volume. On the other hand, it is an exact relation in nonrelativistic models including the 1D Bose gas and the Heisenberg spin chains. Similar theorems had been known for particular cases in the ABA literature [23]. Moreover, after our work was finished, we were informed that a proof of this theorem applicable to spin chains was already given in Ref. [62]; see Sec. VII there. Nevertheless, we present our own proof in Sec. VI, which uses different methods.

In Ref. [63], it was shown that the LeClair-Mussardo (LM) formula (an integral series developed for thermal mean values) can be considered a thermodynamic limit of this expansion theorem, whereas in Ref. [64], it was shown that alternatively Eq. [61] can be derived from the LM formula using certain analytic continuations. In Ref. [65], it was also shown that in IQFT it can be derived directly using the off-diagonal relation [60]. Regarding the continuum gas models, the expansion was proven for certain local operators in the Lieb-Liniger models in Ref. [66].

We note that there is an alternative expansion theorem using the connected form factors [61], but we do not use it here.

The continuity relations yield a connection between the symmetric form factors of the charge and current operators. Introducing the shorthand notations

$$
\begin{aligned}
& \mathcal{Q}_{\alpha}\left(\{\lambda\}_{N}\right) \equiv F_{s}^{Q_{\alpha}(0)}\left(\{\lambda\}_{N}\right), \\
& \mathcal{J}_{\alpha}\left(\{\lambda\}_{N}\right) \equiv F_{s}^{J_{\alpha}(0)}\left(\{\lambda\}_{N}\right),
\end{aligned}
$$

we get from Eq. (4.1),

$$
\left(\sum_{j=1}^{N} e^{\prime}\left(\lambda_{j}\right)\right) \mathcal{Q}_{\alpha}\left(\{\lambda\}_{N}\right)=\left(\sum_{j=1}^{N} p^{\prime}\left(\lambda_{j}\right)\right) \mathcal{J}_{\alpha}\left(\{\lambda\}_{N}\right) .
$$

We remark that Eq. (4.1) is defined only for the finitely many eigenstates of a finite-volume chain, whereas Eq. (4.10) holds for arbitrary rapidities, i.e., not only for the solutions to the Bethe equations. The reason lies in the fact that the form factors are physical amplitudes which are independent of the volume; in spin chains they are rational functions of the rapidities [23]. The relation (4.1) holds in any volume; thus, the Bethe states eventually sample Eq. (4.10) at infinitely many points, and this information is enough to obtain the equality of the rational functions. 
Similar arguments can be made even in QFT situations based on the analyticity properties of the form factors. Alternatively, Eq. (4.10) can be obtained directly in infinite volume using the methods of Ref. [59], but we do not pursue this direction here.

Now our strategy is the following: First we find the symmetric form factors of the charge densities by comparing the formula of the expansion Theorem 1 to the known mean values (2.13). Next, we use the above relation to find the $\mathcal{J}_{\alpha}$. Finally, we use Theorem 1 for the current operators: We sum up the resulting expansion to obtain Eq. (2.18). Essentially, the same strategy has already been applied in Refs. $[10,21]$ directly in the thermodynamic limit using the LeClair-Mussardo series. The novelty of our approach is that we perform these steps in finite volume and that we also provide the proof of our expansion theorem for the $X X X$ and $X X Z$ spin chains (see Sec. VI).

\section{A. The form factors of the charge densities}

We consider Eq. [61] in the case of the charge-density operator $Q_{\alpha}(0)$ and write it as

$\frac{\rho(\{\lambda\})}{L} \sum_{j=1}^{N} q_{\alpha}\left(\lambda_{j}\right)=\sum_{\left\{\lambda^{+}\right\} \cup\left\{\lambda^{-}\right\}} \mathcal{Q}_{\alpha}\left(\left\{\lambda^{+}\right\}\right) \rho\left(\left\{\lambda^{-}\right\}\right)$.

These equations are algebraic relations that hold for any particle number $N$ and any finite volume $L$. The symmetric diagonal form factors can be extracted using a recursive procedure: We consider the above algebraic relations for $N=1,2, \ldots$, and at each $N$ we compute the $N$-particle form factor by subtracting the terms with all $\mathcal{Q}_{\alpha}\left(\left\{\lambda^{+}\right\}\right)$with a lower number of particles obtained earlier.

At $N=1$, the relation immediately gives

$$
\mathcal{Q}_{\alpha}(\lambda)=p^{\prime}(\lambda) q_{\alpha}(\lambda),
$$

where we use Eq. (4.5) and substitute $\left\langle Q_{\alpha}(0)\right\rangle=0$.

At $N=2$, we use Eqs. (4.5)-(4.7). Substituting them into Eq. (4.11), and using also Eq. (4.12) we observe the cancellation of some terms, leading eventually to

$$
\mathcal{Q}_{\alpha}\left(\lambda_{1}, \lambda_{2}\right)=\left[q_{\alpha}\left(\lambda_{1}\right)+q_{\alpha}\left(\lambda_{2}\right)\right]\left[p^{\prime}\left(\lambda_{1}\right)+p^{\prime}\left(\lambda_{2}\right)\right] \varphi_{12} .
$$

This procedure does, in principle, yield all higher symmetric form factors. However, the direct recursive subtractions for $N \geq 3$ become more involved, and it is advantageous to use an alternative method. For the computation of the Gaudin determinants, we apply a graph theoretical matrix-tree theorem, which has already proven to be useful for different problems $[21,67,68]$.

Let us introduce the following definitions. Given a graph $\Gamma$, a directed graph $\mathcal{F}$ is a directed spanning forest of $\Gamma$ if it satisfies the following: (i) $\mathcal{F}$ includes all vertices of $\Gamma$.

(ii) $\mathcal{F}$ does not include any circles.

(iii) Each vertex has at most one incoming edge.

The nodes without incoming edges are called roots. Each spanning forest can be decomposed as a union of spanning trees, which are the connected components of the forest. It can be seen from the above definitions that each spanning tree has exactly one root.

Theorem 2 Let $G$ be an $N \times N$ matrix obtained as the difference $G=D-K$, where $D$ is diagonal and $K$ satisfies the property

$$
\sum_{k=1}^{N} K_{j k}=0, \quad j=1, \ldots, N
$$

In this case, the determinant of $G$ can be expressed as a sum over the directed spanning forests of the complete graph with $N$ nodes. For each spanning forest $\mathcal{F}$, let $R(\mathcal{F})$ denote the set of the roots, and let $l_{j k}$ denote the edges of $\mathcal{F}$ pointing from node $j$ to $k$. Then we have

$$
\operatorname{det} G=\sum_{\mathcal{F}} \prod_{j \in R(\mathcal{F})} D_{j j} \prod_{l_{j k} \in \mathcal{F}} K_{j k}
$$

For a proof, see, e.g., Ref. [69].

The Gaudin determinant given by Eq, (2.16) satisfies the requirements of this theorem with $D_{j j}=p^{\prime}\left(\lambda_{j}\right) L$ and $K_{j k}=\varphi\left(\lambda_{j}-\lambda_{k}\right)$. The main idea to obtain the form factors from Eq. (4.11) is to consider the formal $L \rightarrow 0$ limit of this relation. To do this, we need to observe the $L \rightarrow 0$ limit of the various Gaudin determinants. Each term in Eq. (4.15) carries a factor of $L^{r}$ where $r \geq 1$ is the number of roots for the particular spanning forest $\mathcal{F}$. On the rhs of Eq. (4.11) we have

$$
\lim _{L \rightarrow 0} \rho\left(\left\{\lambda^{-}\right\}\right)=0
$$

for every nonempty set of $\left\{\lambda^{-}\right\}$. Thus, the only term on the rhs which survives the $L \rightarrow 0$ limit is the one where $\left\{\lambda_{-}\right\}=\varnothing$ yielding $\mathcal{Q}_{\alpha}(\{\lambda\})$. On the other hand, on the lhs, we need to keep the $\mathcal{O}(L)$ term in $\rho(\{\lambda\})$, which according to the above theorem, gives an expansion over all directed spanning trees $\mathcal{F}^{\prime}$ :

$$
\lim _{L \rightarrow 0} \frac{\rho\left(\{\lambda\}_{N}\right)}{L}=\sum_{\mathcal{F}^{\prime}}\left[p^{\prime}\left(\lambda_{r}\right) \prod_{l_{j k} \in \mathcal{F}^{\prime}} \varphi_{j k}\right],
$$

where for each spanning tree $\mathcal{F}^{\prime}$ the index $r$ denotes its root vertex, and we use the abbreviation $\varphi_{j k}=\varphi\left(\lambda_{j}-\lambda_{k}\right)$.

Each directed spanning tree can be obtained uniquely from a nondirected spanning tree by selecting the singleroot vertex and choosing the directions of the edges accordingly. In our case, the function $\varphi$ is symmetric; 
thus, the direction of the edges does not influence the factors of $\varphi\left(\lambda_{u}-\lambda_{v}\right)$. Each vertex has to be chosen exactly one time as a root, thus, we obtain

$$
\lim _{L \rightarrow 0} \frac{\rho\left(\{\lambda\}_{N}\right)}{L}=\left[\sum_{j=1}^{N} p^{\prime}\left(\lambda_{j}\right)\right] \sum_{\mathcal{T}} \prod_{l_{j k} \in \mathcal{T}} \varphi_{j k},
$$

where the summation runs over the nondirected spanning trees $\mathcal{T}$.

Finally,

$$
\mathcal{Q}_{\alpha}\left(\{\lambda\}_{N}\right)=\left[\sum_{j=1}^{N} p^{\prime}\left(\lambda_{j}\right)\right]\left[\sum_{j=1}^{N} q_{\alpha}\left(\lambda_{j}\right)\right] \sum_{\mathcal{T}} \prod_{l_{j k} \in \mathcal{T}} \varphi_{j k} .
$$

\section{B. Summation for the current operators}

Equations (4.19) and (4.10) yield the symmetric diagonal form factors of the current operators:

$$
\mathcal{J}_{\alpha}\left(\{\lambda\}_{N}\right)=\left[\sum_{j=1}^{N} e^{\prime}\left(\lambda_{j}\right)\right]\left[\sum_{j=1}^{N} q_{\alpha}\left(\lambda_{j}\right)\right] \sum_{\mathcal{T}} \prod_{l_{j k} \in \mathcal{T}} \varphi_{j k} .
$$

Our task is to sum up the expansion for the currents

$$
\left\langle\{\lambda\}_{N}\left|J_{\alpha}(0)\right|\{\lambda\}_{N}\right\rangle=\frac{\sum_{\left\{\lambda^{+}\right\} \cup\left\{\lambda^{-}\right\}} \mathcal{J}_{\alpha}\left(\left\{\lambda^{+}\right\}\right) \rho\left(\lambda^{-}\right)}{\rho(\{\lambda\})} .
$$

Once again, it is instructive to consider the first few cases. At $N=1$, we have

$$
\mathcal{J}_{\alpha}(\lambda)=e^{\prime}(\lambda) q_{\alpha}(\lambda)
$$

Using $\left\langle J_{\alpha}(0)\right\rangle_{0}=0$ and Eq. (4.5) gives immediately

$$
\left\langle\lambda\left|J_{\alpha}(0)\right| \lambda\right\rangle=\frac{1}{L} \frac{e^{\prime}(\lambda)}{p^{\prime}(\lambda)} q_{\alpha}(\lambda),
$$

as anticipated.

At $N=2$, there are three nonzero terms in the summation:

$$
\frac{\mathcal{J}_{\alpha}\left(\lambda_{1}, \lambda_{2}\right)+\mathcal{J}_{\alpha}\left(\lambda_{1}\right) \rho\left(\lambda_{2}\right)+\mathcal{J}_{\alpha}\left(\lambda_{2}\right) \rho\left(\lambda_{1}\right)}{\rho\left(\lambda_{1}, \lambda_{2}\right)} .
$$

The two-particle symmetric form factor is

$\mathcal{J}_{\alpha}\left(\lambda_{1}, \lambda_{2}\right)=\left[e^{\prime}\left(\lambda_{1}\right)+e^{\prime}\left(\lambda_{2}\right)\right]\left[q_{\alpha}\left(\lambda_{1}\right)+q_{\alpha}\left(\lambda_{2}\right)\right] \varphi_{12}$.

Substituting this equation and also Eqs. (4.22) and (4.5) into Eq. (4.24), we can express the mean value in the product form

$$
\frac{\left(e^{\prime}\left(\lambda_{1}\right) e^{\prime}\left(\lambda_{2}\right)\right)\left(\begin{array}{cc}
p^{\prime}\left(\lambda_{2}\right)+\varphi_{12} & \varphi_{12} \\
\varphi_{12} & p^{\prime}\left(\lambda_{1}\right)+\varphi_{12}
\end{array}\right)\left(\begin{array}{l}
q_{\alpha}\left(\lambda_{1}\right) \\
q_{\alpha}\left(\lambda_{2}\right)
\end{array}\right)}{\rho\left(\lambda_{1}, \lambda_{2}\right)} .
$$

We can recognize the inverse of the two-particle Gaudin matrix (4.6). Thus, in this case we obtain

$$
\left\langle\lambda_{1}, \lambda_{2}\left|J_{\alpha}(0)\right| \lambda_{1}, \lambda_{2}\right\rangle=\left(e^{\prime}\left(\lambda_{1}\right) e^{\prime}\left(\lambda_{2}\right)\right) G^{-1}\left(\begin{array}{c}
q_{\alpha}\left(\lambda_{1}\right) \\
q_{\alpha}\left(\lambda_{2}\right)
\end{array}\right),
$$

as we state in our main result (2.18).

The summation for $N \geq 3$ is considerably more involved. From the structure of the form factors and the Gaudin determinants, we can see that for each pair $j k$ of particles there will be various contributions including the factors $e^{\prime}\left(\lambda_{j}\right) q_{\alpha}\left(\lambda_{k}\right)$ stemming from different terms in Eq. (4.21). Our main result (2.18) states that the sum of these terms will reproduce the $j k$ element of the matrix $G^{-1}$. For this inverse matrix, we can use the formula

$$
G^{-1}=\frac{\operatorname{adj}(G)}{\operatorname{det} G},
$$

where $\operatorname{adj}(G)$ is the so-called adjugate matrix of $G$. The Gaudin determinant is present in the denominator of Eq. (4.21); thus, Eq. (2.18) holds if in the nominator the sum of the terms with $e^{\prime}\left(\lambda_{j}\right) q_{\alpha}\left(\lambda_{k}\right)$ reproduce the $j k$ components of $\operatorname{adj}(G)$. The proof of this statement is not trivial, and it is presented in Appendix C.

\section{Mean values of the generalized currents}

It is straightforward to repeat the previous calculations for the case of the generalized currents $J_{\alpha}^{\beta}$ defined in Eq. (2.25). For the symmetric diagonal form factors, we use the notation $\mathcal{J}_{\alpha}^{\beta}\left(\{\lambda\}_{N}\right)$. The local continuity equation then leads to

$$
\left(\sum_{j=1}^{N} q_{\beta}^{\prime}\left(\lambda_{j}\right)\right) \mathcal{Q}_{\alpha}\left(\{\lambda\}_{N}\right)=\left(\sum_{j=1}^{N} p^{\prime}\left(\lambda_{j}\right)\right) \mathcal{J}_{\alpha}^{\beta}\left(\{\lambda\}_{N}\right),
$$

from which

$$
\mathcal{J}_{\alpha}^{\beta}\left(\{\lambda\}_{N}\right)=\left[\sum_{j=1}^{N} q_{\beta}^{\prime}\left(\lambda_{j}\right)\right]\left[\sum_{j=1}^{N} q_{\alpha}\left(\lambda_{j}\right)\right] \sum_{\mathcal{T}} \prod_{l_{j k} \in \mathcal{T}} \varphi_{j k} .
$$

These form factors are needed to sum up the expansion theorem [61]. All of the previous computations can be 
applied by exchanging the function $e^{\prime}(\lambda)$ with $q_{\beta}^{\prime}(\lambda)$. This change has a simple interpretation: For the general currents, the time evolution is dictated by $Q_{\beta}$ instead of the physical Hamiltonian. Performing all the previous steps, we obtain our result (2.26).

\section{CURRENT OPERATORS AND ALGEBRAIC BETHE ANSATZ IN INTEGRABLE LATTICE MODELS}

Here we review the canonical construction of the conserved charge and associated current operators; we also sketch the standard method of the algebraic Bethe ansatz to find the eigenstates. We concentrate on lattice models that are obtained from integrable Lax operators. The treatment below is rather general, with concrete examples given later in Sec. V B.

Let $\mathcal{H}=V_{1} \otimes V_{2} \otimes \ldots \otimes V_{L}$ denote the Hilbert space of the model, with $V_{j} \approx \mathbb{C}^{D}$ with some $D=2,3, \ldots$, and let $V_{a} \approx \mathbb{C}^{\tilde{D}}$ with some $\tilde{D}$ denote the so-called auxiliary space. In our main examples, $\tilde{D}=D$, but this is not necessary. Let $\mathcal{L}(u)$ denote the so-called Lax operator acting on $V_{j} \otimes V_{a}$. Here, $u \in \mathbb{C}$ is the spectral parameter.

The monodromy matrix $T(u)$ acting on $V_{a} \otimes \mathcal{H}$ is defined as

$$
T(u)=\mathcal{L}_{1, a}(u), \ldots, \mathcal{L}_{L, a}(u)
$$

The transfer matrix is given by the trace in auxiliary space, which corresponds to enforcing periodic boundary conditions:

$$
t(u)=\operatorname{Tr}_{a} T(u)
$$

The local Lax operators satisfy the exchange property

$$
\mathcal{L}_{1 a}(u) \mathcal{L}_{1 b}(v) R_{a b}(v-u)=R_{a b}(v-u) \mathcal{L}_{1 b}(v) \mathcal{L}_{1 a}(u),
$$

where we introduce two auxiliary spaces $V_{a, b}$, and $R(u)$ is the so-called $R$ matrix acting on $V_{a} \otimes V_{b}$. It satisfies the Yang-Baxter relation

$$
\begin{aligned}
& R_{12}\left(u_{1}-u_{2}\right) R_{13}\left(u_{1}-u_{3}\right) R_{23}\left(u_{2}-u_{3}\right) \\
& \quad=R_{23}\left(u_{2}-u_{3}\right) R_{13}\left(u_{1}-u_{3}\right) R_{12}\left(u_{1}-u_{2}\right),
\end{aligned}
$$

which is a relation of operators acting on the triple product $V_{1} \otimes V_{2} \otimes V_{3}$ of auxiliary spaces.

As an effect of these relations, the transfer matrices form a commuting family of operators [23]:

$$
[t(u), t(v)]=0 .
$$

The transfer matrix encodes the hierarchy of the conserved charges, which are obtained by expanding $t(u)$ around certain special points.

We consider models where the dimensions of the physical and auxiliary spaces are equal, and the local Lax operator can be chosen to be identical to the $R$ matrix: $\mathcal{L}(u)=R(u)$. Furthermore, we consider cases where the $R$ matrix satisfies the initial condition $R(0)=P$ with $P$ being the permutation operator, such that the transfer matrix satisfies

$$
t(0)=U,
$$

where $U$ is the translation operator by one site.

For any $\alpha \in \mathbb{N}, \alpha \geq 2$, we then define

$$
Q_{\alpha}=\left.i\left(\frac{\partial}{\partial u}\right)^{\alpha-1} \log [t(u)]\right|_{u=0} .
$$

These charges will be extensive, and they can be written as a sum over the charge-density operators [70]:

$$
Q_{\alpha}=\sum_{j=1}^{L} Q_{\alpha}(x) .
$$

With this definition, $Q_{\alpha}(x)$ spans $\alpha$ sites, and for $\alpha=2$ we have

$$
Q_{2}=\kappa H,
$$

with $H$ being the physical Hamiltonian and $\kappa$ being an $L$-independent factor, which depends on the particular conventions that are used. If the spectral parameter is chosen appropriately, then the canonical $Q_{\alpha}$ defined above are all Hermitian.

We consider models with particle number conservation. In these cases, there is a reference state $|0\rangle$ with zero particles present. We require that the overall normalization of the transfer matrix satisfies

$$
\langle 0|t(u)| 0\rangle=1,
$$

leading to

$$
\left\langle 0\left|Q_{\alpha}\right| 0\right\rangle=0 .
$$

Additional multiplicative factors in $t(u)$ will alter the additive normalization of the charges.

Having found the charge densities, the continuity relations (2.5) and (2.25) uniquely define the current and generalized current operators $J_{\alpha}$ and $J_{\alpha}^{\beta}$. It follows from Eq. (5.9) that for $\beta=2$ we can identify $J_{\alpha}^{2}=\kappa J_{\alpha}$.

We note that the relations (5.7) and (5.8) do not define $Q_{\alpha}(x)$ unambiguously: For any choice $Q_{\alpha}(x)$, we can take a local operator $D(x)$ and define an alternative density 


$$
Q_{\alpha}^{\prime}(x)=Q_{\alpha}(x)+D(x+1)-D(x),
$$

which leads to the same integrated charge. This transformation can be considered as a "gauge freedom" for the definition of the charge densities, and it is discussed in detail in Ref. [19]. This "gauge choice" does not alter the charge mean values, but it changes the definition of the current operators as

$$
J_{\alpha}^{\beta^{\prime}}(x)=J_{\alpha}^{\beta}(x)-i\left[Q_{\beta}, D(x)\right] .
$$

The additional terms do not affect the mean values of $Q_{\alpha}(x)$ and $J_{\alpha}^{\beta}(x)$.

An alternative way of constructing the charges is with the help of the boost operator [71-74], which is defined on the infinite chain as the formal expression

$$
B=\sum_{x=-\infty}^{\infty} x Q_{2}(x)
$$

where the density of the charge $Q_{2}$ is simply

$$
Q_{2}(x)=\left.i P_{x, x+1} \frac{d R_{x, x+1}(\lambda)}{d \lambda}\right|_{\lambda=0} .
$$

A formal manipulation shows that $[73,74]$

$$
\frac{d t_{\infty}(\lambda)}{d \lambda}=i\left[B, t_{\infty}(\lambda)\right]+\text { const },
$$

where $t_{\infty}(\lambda)$ is the transfer matrix of an infinite chain. It follows that

$$
Q_{\alpha+1}=i\left[B, Q_{\alpha}\right]+\text { const. }
$$

The additional constant parts are not fixed by the formal computations and need to be adjusted afterward. Defining a recursion as $\tilde{Q}_{\alpha+1}=i\left[B, Q_{\alpha}\right]$ and using Eq. (5.11), we get the correct normalization

$$
Q_{\alpha+1}=\tilde{Q}_{\alpha+1}-\left\langle 0\left|\tilde{Q}_{\alpha+1}\right| 0\right\rangle .
$$

It is also possible to compute the current operators $J_{\alpha}^{\beta}(x)$ using a generalization of the boost operator. A formal application of Eq. (2.25) gives

$$
i\left[Q_{\beta}, \sum_{x} x Q_{\alpha}(x)\right]=\sum_{x} J_{\alpha}^{\beta}(x) .
$$

This relation can be used to obtain $J_{\alpha}^{\beta}(x)$, but depending on the situation the direct application of Eq. (2.25) might be more efficient.

\section{A. The Bethe ansatz solution}

Let us now focus on the case of $D=\bar{D}=2$. Furthermore, we consider models with $U(1)$ symmetry where the fundamental $R$ matrix is of the form

$$
R(u)=\left(\begin{array}{cccc}
1 & 0 & 0 & 0 \\
0 & b(u) & c(u) & 0 \\
0 & c(u) & b(u) & 0 \\
0 & 0 & 0 & 1
\end{array}\right)
$$

Specific examples are given later in Sec. V B.

The monodromy matrix defined in Eq. (5.1) is usually written in the block form

$$
T(u)=\left(\begin{array}{ll}
A(u) & B(u) \\
C(u) & D(u)
\end{array}\right),
$$

where the blocks correspond to the degrees of freedom in auxiliary space, and $A(u), B(u), C(u), D(u)$ are operators acting on the spin chain.

It follows from the local relation (5.3) that the monodromy matrix satisfies

$T_{a}(u) T_{b}(v) R_{a b}(v-u)=R_{a b}(v-u) T_{b}(v) T_{a}(u)$,

where $a, b$ refer to two different auxiliary spaces. Commutation relations between the $A, B, C, D$ operators can be derived from Eq. (5.22); they are listed in Appendix D.

In our models possessing $U(1)$ symmetry, there exists a reference state $|0\rangle$ which is annihilated by all $C(u)$ for all $u$. Typically, $|0\rangle$ is chosen as the state with all spins up. Then, the Bethe states can be created in an algebraic Bethe ansatz as

$$
\left|\lambda_{1}, \ldots, \lambda_{N}\right\rangle=\prod_{j=1}^{N} B\left(\lambda_{j}-\sigma\right)|0\rangle .
$$

Here, $\sigma$ is a constant which is chosen later such that the rapidity parametrization becomes exactly the same as in the coordinate Bethe ansatz.

These states are eigenstates of the spin chain Hamiltonian if the rapidities satisfy the Bethe equations

$$
\frac{a\left(\lambda_{j}-\sigma\right)}{d\left(\lambda_{j}-\sigma\right)} \prod_{k \neq j} \frac{f\left(\lambda_{k}, \lambda_{j}\right)}{f\left(\lambda_{j}, \lambda_{k}\right)}=1,
$$

where we introduce the function

$$
f(u, v)=\frac{1}{b(u-v)},
$$

and the vacuum eigenvalues $a(u), d(u)$ defined as 


$$
A(u)|0\rangle=a(u)|0\rangle, \quad D(u)|0\rangle=d(u)|0\rangle .
$$

For $R$ matrices of the form (5.20), we have

$$
a(u)=1, \quad d(u)=[b(u)]^{L} .
$$

Dual eigenstates are created as

$$
\left\langle\lambda_{1}, \ldots, \lambda_{N}\right|=\langle 0| \prod_{j=1}^{N} C\left(\lambda_{j}-\sigma\right) .
$$

For on-shell states of the physical chain, these states are the adjoints of the states (5.23) (for a detailed proof, see Ref. [23]), but in the more general case, including certain inhomogeneity parameters (leading to non-Hermitian Hamiltonians), they are only dual vectors.

For eigenvectors, the norm of the Bethe states is

$$
\begin{aligned}
\langle 0| & \prod_{j=1}^{N} C\left(\lambda_{j}\right) \prod_{j=1}^{N} B\left(\lambda_{j}\right)|0\rangle \\
& =(\tilde{\kappa})^{N}\left(\prod_{j \neq k} f\left(\lambda_{j}, \lambda_{k}\right)\right) \operatorname{det} G_{\lambda},
\end{aligned}
$$

where $\tilde{\kappa}$ is a model-dependent constant. This statement was proven in Ref. [48] based on the singularity properties of general overlaps.

Finally, the eigenvalues of the transfer matrix are

$$
t(u)=a(u) \prod_{j=1}^{N} f\left(\lambda_{j}-\sigma, u\right)+d(u) \prod_{j=1}^{N} f\left(u, \lambda_{j}-\sigma\right) .
$$

\section{B. Main examples}

The $S U(2)$-invariant $X X X$ Heisenberg spin chain is given by the Hamiltonian

$$
H=\sum_{j=1}^{L}\left(\sigma_{j}^{x} \sigma_{j+1}^{x}+\sigma_{j}^{y} \sigma_{j+1}^{y}+\sigma_{j}^{z} \sigma_{j+1}^{z}-1\right),
$$

where $\sigma_{j}^{a}, a=x, y, z$ are the Pauli matrices acting on site $j$. This integrable model can be obtained from the $R$ matrix of the form (5.20) with

$$
b(u)=\frac{u}{u+i}, \quad c(u)=\frac{i}{u+i},
$$

where the shift parameter is $\sigma=i / 2$ and $\tilde{\kappa}=1$.

The canonical definition (5.7) of the charges gives $Q_{2}=H / 2$ [23]; the additive normalization is such that Eq. (5.11) is satisfied.

The eigenstates of the model organize themselves into $S U(2)$ multiplets, and the Bethe ansatz gives the highest weight vectors. The wave functions are of the form (2.8) with the rapidity parametrization given by

$$
\begin{aligned}
e^{i p(\lambda)} & =\frac{\lambda-i / 2}{\lambda+i / 2}, \\
e^{i \delta(\lambda)} & =\frac{\lambda+i}{\lambda-i} .
\end{aligned}
$$

The one-particle energy eigenvalue is $e(\lambda)=2 q_{2}(\lambda)$ with

$$
q_{2}(\lambda)=-p^{\prime}(\lambda)=-\frac{1}{\lambda^{2}+1 / 4}
$$

The rapidity parameters take values in the whole complex plane. The solutions of the Bethe equations organize themselves into strings, which describe bound states of spin waves [49]. These bound states can be regarded as different particle types in the thermodynamic limit. However, we perform our finite-volume analysis on the level of the individual Bethe roots; therefore, we do not treat the string solutions separately.

In this model, the canonical charges $Q_{\alpha}$ defined by Eq. (5.7) can be computed explicitly [75]. For the sake of completeness, we present the explicit formulas up to $Q_{4}$ in Appendix B; more explicit results are found in Ref. [75].

Regarding the one-particle eigenvalues of the charges, it follows directly from Eq. (5.30) that

$$
q_{\alpha}(\lambda)=\left.\left(\frac{\partial}{\partial x}\right)^{\alpha-2} q_{2}(\lambda-x)\right|_{x=0} .
$$

The transfer matrix commutes with the global $S U(2)$ transformations; therefore, the $Q_{\alpha}$ with $\alpha \geq 2$ are all $S U$ (2)-invariant operators. The global spin operators are additional conserved quantities, and the traditional choice is to add $Q_{1}=S^{z}$ into the commuting family. If the vacuum is chosen as the reference state with all spins up, then the one-particle eigenvalues of $Q_{1}$ are simply $q_{1}(\lambda)=-1$.

Explicit real-space formulas for the current operators of the $X X X$ model are not available. Based on Ref. [75], it seems plausible that closed-form results can be computed, but we do not pursue this direction. Nevertheless, we compute the first few currents and generalized currents; the results are presented in Sec. B.

Our second example is the $X X Z$ model, which is given by the Hamiltonian

$$
H=\sum_{j=1}^{L}\left[\sigma_{j}^{x} \sigma_{j+1}^{x}+\sigma_{j}^{y} \sigma_{j+1}^{y}+\Delta\left(\sigma_{j}^{z} \sigma_{j+1}^{z}-1\right)\right] .
$$

This model can be obtained from an $R$ matrix of the form (5.20) with 


$$
b(u)=\frac{\sin (u)}{\sin (u+i \eta)}, \quad c(u)=\frac{\sin (i \eta)}{\sin (u+i \eta)},
$$

where $\Delta=\cosh (\eta)$. In this normalization, we have $Q_{2}=$ $H /(2 \sinh \eta)$. The shift parameter is $i \eta / 2$, and $\tilde{\kappa}=\sinh (\eta)$.

For simplicity, we focus on the regime $\Delta>1$, where the rapidity parameters take values in the strip $\Re(\lambda) \in$ $[-\pi / 2, \pi / 2]$, and the momentum and scattering amplitudes are

$$
\begin{aligned}
e^{i p(\lambda)} & =\frac{\sin (\lambda-i \eta / 2)}{\sin (\lambda+i \eta / 2)}, \\
e^{i \delta(\lambda)} & =\frac{\sin (\lambda+i \eta)}{\sin (\lambda-i \eta)},
\end{aligned}
$$

with the one-particle energy being $e(\lambda)=2 \sinh (\eta) q_{2}(\lambda)$, where now

$$
q_{2}(\lambda)=\frac{\sinh (\eta)}{2[\cos (2 \lambda)-\cosh (\eta)]} .
$$

The one-particle eigenvalues of the canonical charges $Q_{\alpha}$ are again given by Eq. (5.35) with the $q_{2}(\lambda)$ function above. The real-space representation of the $Q_{\alpha}$ is treated in detail in Ref. [74], but we do not use those results here. The $U(1)$ invariance of the model leads to the independent conserved charge $Q_{1}=S^{z}$ with one-particle eigenvalues $q_{1}(\lambda)=-1$.

\section{PROOF OF THE EXPANSION THEOREM: THE $X X Z$ CHAIN}

Here we prove Theorem 1 using standard methods of the ABA [23]. In fact, our proof can be considered a generalization of the proof given by Korepin for the norms of the Bethe ansatz wave functions [48]. Similar ideas have been worked out by one of the authors in the work [63], which considered certain local correlators of the continuum 1D Bose gas. Here we restrict ourselves to the case of the $X X Z$ spin chain, with the $R$ matrix given by conventions (5.20)-(5.37). The case of the $X X X$ chain can be treated similarly.

For the proof, it is useful to define renormalized operators

$$
\mathbb{B}(u)=\frac{B(u)}{d(u)}, \quad \mathbb{C}(u)=\frac{C(u)}{d(u)} .
$$

In order to shorten the notations, in this section we do not use the rapidity shift $-i \eta / 2$ that appears in Eq. (5.23).

Our aim is to derive the form-factor expansion for the normalized mean values

$$
\frac{\left\langle 0\left|\prod_{j=1}^{N} \mathbb{C}\left(\lambda_{j}\right) \mathcal{O} \prod_{j=1}^{N} \mathbb{B}\left(\lambda_{j}\right)\right| 0\right\rangle}{\left\langle 0\left|\prod_{j=1}^{N} \mathbb{C}\left(\lambda_{j}\right) \prod_{j=1}^{N} \mathbb{B}\left(\lambda_{j}\right)\right| 0\right\rangle},
$$

where $\mathcal{O}$ is any operator of the finite spin chain. Quite interestingly, for this proof we do not require any locality property from the operator. In fact, locality of an operator is not even a well-defined concept in a finite chain. Thus, we do not impose any restriction on the range of the operator $\mathcal{O}$.

To be precise, let us consider the elementary matrices $E_{a b}, a, b=1,2$ and let $E_{a b}(x)$ stand for operator which acts as $E_{a b}$ on site $x=1, \ldots, L$ and with the identity elsewhere. We perform the proof for an arbitrary product

$$
\mathcal{O}=\prod_{x=1}^{L} E_{a_{x} b_{x}}(x) .
$$

Each operator of the finite chain is a linear combination of these products. Short-range operators are obtained by taking traces in some subset of the indices $a_{x}, b_{x}$.

In order to compute these mean values, we need to embed the operators (6.3) in the Yang-Baxter algebra. This procedure is called the "quantum-inverse-scattering problem" and was solved in Refs. [76-78].

In the case of the homogeneous chain, we have

$$
\prod_{x=L}^{1} E_{a_{x} b_{x}}(x)=\prod_{x=L}^{1} T_{a_{x} b_{x}}(0) .
$$

Our strategy is that we prove the theorem for an arbitrary product

$$
\mathcal{O}=X\left(\mu_{L}\right), \ldots, X\left(\mu_{2}\right) X\left(\mu_{1}\right),
$$

where $X(\mu)$ may represent one of the four operators $A, B$, $C, D$ evaluated at spectral parameter $\mu$. Afterward, we take the $\mu_{j} \rightarrow 0$ limit, and by continuity we obtain the statement for Eq. (6.4).

Our proof is based on the singularity properties of the matrix elements of operators. We follow closely the proof of Korepin for the norms of Bethe states [48]; in fact, our proof can be considered a slight generalization of the methods of Korepin. For an earlier similar proof, see Ref. [63].

We introduce the following notation for a matrix element of the arbitrary operator $\mathcal{O}$ between two states (not necessarily eigenstates) described by the rapidity sets $\left\{\lambda^{B}\right\}_{N}$ and $\left\{\lambda^{C}\right\}_{N}$,

$$
\begin{array}{r}
M_{N}^{\mathcal{O}}\left(\left\{\lambda^{C}\right\}_{N},\left\{\lambda^{B}\right\}_{N},\left\{l^{C}\right\}_{N},\left\{l^{B}\right\}_{N}\right) \\
\quad=\left\langle 0\left|\prod_{j=1}^{N} \mathbb{C}\left(\lambda_{j}^{C}\right) \mathcal{O} \prod_{j=1}^{N} \mathbb{B}\left(\lambda_{j}^{B}\right)\right| 0\right\rangle .
\end{array}
$$

These matrix elements depend on the rapidities and the variables $l(\lambda)=a(\lambda) / d(\lambda)$, where $a(\lambda)$ and $d(\lambda)$ are the vacuum expectation values of the operators $A(\lambda)$ and $D(\lambda)$, 
respectively [23]. For what follows, it is important that we can consider arbitrary functions for $a(\lambda)$ and $d(\lambda)$, and therefore, $M_{N}^{\mathcal{O}}$ is the function of $4 N$ independent variables. It follows from the commutativity of the $\mathbb{B}$ and $\mathbb{C}$ operators that $M_{N}^{\mathcal{O}}$ is invariant with respect to simultaneous exchanges $\lambda_{j}^{B} \leftrightarrow \lambda_{k}^{B}, l_{j}^{B} \leftrightarrow l_{k}^{B}$, and similar for the rapidities on the lefthand side.

The matrix elements have apparent singularities as two rapidities from the two sides approach each other. These apparent poles result from the commutation relations between the $\mathbb{B}$ and $\mathbb{C}$ operators. We show that the structure of these poles completely determines the mean values. For simplicity, we focus on the singularities as $\lambda_{N}^{C}-\lambda_{N}^{B} \rightarrow 0$; the other cases follow simply from the permutation symmetry.

Theorem 3. The matrix elements satisfy the following singularity property:

$$
\begin{aligned}
& M_{N}^{\mathcal{O}}\left(\left\{\lambda^{C}\right\}_{N},\left\{\lambda^{B}\right\}_{N},\left\{l^{C}\right\}_{N},\left\{l^{B}\right\}_{N}\right) \stackrel{\lambda_{N}^{C} \rightarrow \lambda_{N}^{B}}{\longrightarrow} \\
& \rightarrow \frac{i \sinh (\eta)}{\lambda_{N}^{C}-\lambda_{N}^{B}}\left(l_{N}^{C}-l_{N}^{B}\right)\left(\prod_{k=1}^{N-1} f_{k N}^{C} f_{k N}^{B}\right) \\
& \quad \times M_{N-1}^{\mathcal{O}}\left(\left\{\lambda^{C}\right\}_{N-1},\left\{\lambda^{B}\right\}_{N-1},\left\{l_{\text {mod }}^{C}\right\}_{N-1},\left\{l_{\text {mod }}^{B}\right\}_{N-1}\right),
\end{aligned}
$$

where in the third line the matrix element is calculated with the modified vacuum expectation values

$$
a_{j, \text { mod }}=a_{j} f\left(\lambda_{N}, \lambda_{j}\right), \quad d_{j, \text { mod }}=d_{j} f\left(\lambda_{j}, \lambda_{N}\right),
$$

leading to

$$
l_{j, \text { mod }}=l_{j} \frac{f\left(\lambda_{N}, \lambda_{j}\right)}{f\left(\lambda_{j}, \lambda_{N}\right)} .
$$

The proof is rather technical, and it is presented in Appendix D.

In the physical case (when the $l_{N}^{B, C}$ variables are not independent), the prefactor in the previous equation behaves as

$$
\frac{i \sinh (\eta)}{\lambda_{N}^{C}-\lambda_{N}^{B}}\left(l_{N}^{C}-l_{N}^{B}\right) \stackrel{\lambda_{N}^{C} \rightarrow \lambda_{N}^{B}}{\longrightarrow} \sinh (\eta) l(\lambda) z(\lambda),
$$

where $z(\lambda)=i \partial_{\lambda} \log l(\lambda)$. This observation is used to study the diagonal limit.

The diagonal evaluation of the matrix element is defined as

$$
\begin{aligned}
& M_{N, d}^{\mathcal{O}}\left(\{\lambda\}_{N},\{l\}_{N},\{z\}_{N}\right) \\
& \quad=\lim _{\lambda_{k}^{C} \rightarrow \lambda_{k}^{B}} M_{N}^{\mathcal{O}}\left(\left\{\lambda^{C}\right\}_{N},\left\{\lambda^{B}\right\}_{N},\left\{l^{C}\right\}_{N},\left\{l^{B}\right\}_{N}\right),
\end{aligned}
$$

where the limit is performed for every $k=1, \ldots, N$. This quantity depends on $3 N$ independent variables $\{\lambda\}_{N},\{l\}_{N}$, and $\{z\}_{N}$. From Eq. (6.7), it follows that the dependence on $z_{N}$ is linear, and the proportionality factor is

$$
\begin{aligned}
\frac{\partial}{\partial z_{N}} M_{N, d}^{\mathcal{O}}\left(\{\lambda\}_{N},\{l\}_{N},\{z\}_{N}\right) \\
=\sinh (\eta) l\left(\lambda_{N}\right)\left(\prod_{k=1}^{N-1} f_{k N}^{C} f_{k N}^{B}\right) \\
\quad \times M_{N-1, d}^{\mathcal{O}}\left(\{\lambda\}_{N-1},\left\{l_{\bmod }\right\}_{N-1},\left\{z_{\bmod }\right\}_{N-1}\right),
\end{aligned}
$$

where

$$
z_{\bmod }(\lambda)=z(\lambda)+\varphi\left(\lambda-\lambda_{N}\right) .
$$

To calculate the expectation values in the eigenstates of the system, we have to take the rapidities to the solutions of the Bethe equations. In practice, this means that for the $l$ parameters we substitute the ratios of $S$ functions from the Bethe equations (2.11):

$$
\langle\mathcal{O}\rangle_{N}\left(\{\lambda\}_{N},\{z\}_{N}\right)=\left.M_{N, d}^{\mathcal{O}}\left(\{\lambda\}_{N},\{l\}_{N},\{z\}_{N}\right)\right|_{\{l\}_{N} \text { substituted }} .
$$

The dependence on $z_{N}$ is still linear:

$$
\begin{aligned}
& \frac{\partial\langle\mathcal{O}\rangle_{N}\left(\{\lambda\}_{N},\{z\}_{N}\right)}{\partial z_{N}} \\
& =\left(\prod_{k=1}^{N-1} f_{k N} f_{N k}\right) \times \sinh (\eta)\langle\mathcal{O}\rangle_{N-1}\left(\{\lambda\}_{N-1},\left\{z_{\bmod }\right\} N-1\right) .
\end{aligned}
$$

To continue the calculation, we need to introduce the form factors $\mathbb{F}_{N}^{\mathcal{O}}$ and $\mathbb{F}_{N, S}^{\mathcal{O}}$ (these differ from the previously used form factors only in an overall normalization)

$\mathbb{F}_{N}^{\mathcal{O}}\left(\left\{\lambda^{C}\right\}_{N},\left\{\lambda^{B}\right\}_{N}\right)=\left.M_{N}^{\mathcal{O}}\left(\left\{\lambda^{C}\right\}_{N},\left\{\lambda^{B}\right\}_{N},\left\{l^{C}\right\}_{N},\left\{l^{B}\right\}_{N}\right)\right|_{\mathrm{BE}}$,

where it is understood that both sets of rapidities solve the Bethe equations; i.e., we express the $\left\{l^{C}\right\}_{N},\left\{l^{B}\right\}_{N}$ using the rapidities. From Eq. (6.7), it is obvious that this form factor satisfies the following recursion relation:

$$
\begin{aligned}
& \mathbb{F}_{N}^{\mathcal{O}}\left(\left\{\lambda^{C}\right\}_{N},\left\{\lambda^{B}\right\}_{N}\right) \stackrel{\lambda_{N}^{C} \rightarrow \lambda_{N}^{B}}{\longrightarrow} \\
& \rightarrow \frac{i \sinh (\eta)}{\lambda_{N}^{C}-\lambda_{N}^{B}}\left(\prod_{k=1}^{N-1} f_{N k}^{C} f_{k N}^{B}-\prod_{k=1}^{N-1} f_{k N}^{C} f_{N k}^{B}\right) \\
& \quad \times \mathbb{F}_{N-1}^{\mathcal{O}}\left(\left\{\lambda^{C}\right\}_{N-1},\left\{\lambda^{B}\right\}_{N-1}\right) .
\end{aligned}
$$


By taking the symmetric diagonal limit of this quantity, one obtains the symmetric form factor

$$
\mathbb{F}_{N, s}^{\mathcal{O}}\left(\{\lambda\}_{N}\right)=\lim _{\epsilon \rightarrow 0} \mathbb{F}_{N}^{\mathcal{O}}\left(\{\lambda+\epsilon\}_{N},\{\lambda\}_{N}\right) .
$$

Theorem 4. The symmetric form factor of the operator $\mathcal{O}$ is equal to its expectation value in the case where every $z_{j}$ is zero:

$$
\mathbb{F}_{N, S}^{\mathcal{O}}\left(\{\lambda\}_{N}\right)=\langle\mathcal{O}\rangle_{N}\left(\{\lambda\}_{N},\{0\}_{N}\right) .
$$

Proof.-From Eq. (6.7), it is obvious that the $z$ dependence of the expectation value arises from the rapidity dependence of the $l(\lambda)$ function. This means that the $z$-independent irreducible part can be obtained by choosing $l\left(\lambda_{j}^{C}\right)=l\left(\lambda_{j}^{B}\right)$, where $\left\{l^{B}\right\}_{N}$ solves the Bethe equations:

$$
\begin{aligned}
& \langle\mathcal{O}\rangle_{N}\left(\{\lambda\}_{N},\{0\}_{N}\right) \\
& \quad=\lim _{\epsilon \rightarrow 0} M_{N}^{\mathcal{O}}\left(\left\{\lambda^{B}+\epsilon\right\}_{N},\left\{\lambda^{B}\right\}_{N},\left\{l^{B}\right\}_{N},\left\{l^{B}\right\}_{N}\right) .
\end{aligned}
$$

On the other hand, the symmetric form factor is by definition

$\mathbb{F}_{N, s}^{\mathcal{O}}\left(\{\lambda\}_{N}\right)=\lim _{\epsilon \rightarrow 0} M_{N}^{\mathcal{O}}\left(\left\{\lambda^{B}+\epsilon\right\}_{N},\left\{\lambda^{B}\right\}_{N},\left\{\tilde{l}^{B}\right\}_{N},\left\{l^{B}\right\}_{N}\right)$, where both $\left\{l^{B}\right\}_{N}$ and $\left\{\tilde{l}^{B}\right\}_{N}$ solve the Bethe equations. But this means that the elements of $\left\{l^{B}\right\}_{N}$ and $\left\{\tilde{l}^{B}\right\}_{N}$ are the products of the appropriate $S$ matrices, so

$$
\left\{l^{B}\right\}_{N}=\left\{\tilde{l}^{B}\right\}_{N}
$$

This completes the proof.

We define the $S$ function for an arbitrary bipartition of the set $\{\lambda\}_{N}=\left\{\lambda^{+}\right\}_{n} \cup\left\{\lambda^{-}\right\}_{N-n}$ in the following way:

$$
\begin{aligned}
S_{N}\left(\left\{\lambda^{+}\right\}_{n},\left\{\lambda^{-}\right\}_{N-n},\left\{z^{-}\right\}_{N-n}\right) \\
=[\sinh (\eta)]^{N-n} \times\left(\prod_{\lambda_{j} \in\left\{\lambda^{+}\right\}} \prod_{\lambda_{k} \in\left\{\lambda^{-}\right\}} f_{j k}^{+-} f_{k j}^{-+}\right) \\
\quad \times\left(\prod_{1 \leq j<k \leq n} f_{j k}^{--} f_{k j}^{--}\right) \rho\left(\left\{\lambda^{-}\right\}_{N-n},\left\{z^{-}\right\}_{N-n}\right),
\end{aligned}
$$

where $f_{j k}^{+-}=f\left(\lambda_{j}^{+}, \lambda_{k}^{-}\right)$. This function depends on $z_{N}$ only through the Gaudin determinant, and its dependence can be calculated easily by expanding the determinant with respect to its $N$ th row or column. Doing so, one obtains that

$\frac{1}{\sinh (\eta)} \frac{\partial}{\partial z_{N}} S_{N}\left(\left\{\lambda^{+}\right\}_{n},\left\{\lambda^{-}\right\}_{N-n},\{z\}_{N}\right)= \begin{cases}\left(\prod_{k=1}^{N-1} f_{k N} f_{N k}\right) S_{N-1}\left(\left\{\lambda^{+}\right\}_{n},\left\{\lambda^{-}\right\}_{N-n-1},\left\{z_{\text {mod }}^{-}\right\}_{N-n-1}\right) & \lambda_{N} \in\left\{\lambda^{-}\right\}, \\ 0 & \lambda_{N} \in\left\{\lambda^{+}\right\} .\end{cases}$

It is important to notice that if we take all the z's in the argument of $S$ to zero, we get zero:

$$
S_{N}\left(\left\{\lambda^{+}\right\}_{n},\left\{\lambda^{-}\right\}_{N-n},\{0\}_{N-n}\right)=0 .
$$

This result follows from the fact that in this case the Gaudin determinant is zero, which follows from Theorem 2.

With the help of the relations listed above, we are now in the position to prove the following theorem:

Theorem 5 The unnormalized mean value of an arbitrary operator $\mathcal{O}$ in any eigenstate of the system can be calculated in the following way:

$$
\langle\mathcal{O}\rangle_{N}\left(\{\lambda\}_{N}\right)=\sum_{\left\{\lambda^{+}\right\} \cup\left\{\lambda^{-}\right\}} \mathbb{F}_{S}^{\mathcal{O}}\left(\left\{\lambda^{+}\right\}\right) S_{N}\left(\left\{\lambda^{+}\right\},\left\{\lambda^{-}\right\},\left\{z^{-}\right\}\right),
$$

where the summation goes over every bipartition of the set of rapidities $\{\lambda\}_{N}$, and for simplicity we do not denote the cardinality of the sets $\left\{\lambda^{ \pm}\right\}$separately.
Proof.-We use induction in the particle number $N$. Let us consider both sides as the multilinear function of the $z_{j}$ variables. Our goal is to show that both sides depend the same way on every $z_{j}$ and that their $z$-independent parts are also equal. We look at the first $N$ for which the matrix element defined by Eq. (6.6) is not zero, and we denote this number by $N_{\min }$. In the case when $N<N_{\min }$, both sides of Eq. (6.26) are zero; therefore, the equation is satisfied. If $N=N_{\min }$, there is only one nonzero term in the summation on the rhs, namely, when $\left\{\lambda^{+}\right\}=\{\lambda\}_{N}$. This means that on the rhs, there is only $\mathbb{F}_{N, s}^{\mathcal{O}}\left(\{\lambda\}_{N}\right)$, since $S\left(\left\{\lambda^{+}\right\}, \varnothing, \varnothing\right)=1$; therefore, it is $z$ independent. The lhs is also independent of $z$, which follows from Eq. (6.15), but the $z$-independent parts are equal according to the previously proved theorem. Now let us suppose that Eq. (6.26) is satisfied for every $N<M$ and examine the $N=M$ case. On the rhs, only those terms depend on $z_{j}$ where $z_{j} \in\left\{\lambda^{-}\right\}$. By taking the partial derivative of it with respect to $z_{j}$, the initial summation is getting modified to a new one, going over all the bipartitions of the set $\{\lambda\}_{N-1}=\{\lambda\}_{N} \backslash\left\{\lambda_{j}\right\}$ [this statement follows from Eq. (6.24)]. According to the induction assumption, this sum 
gives exactly $\langle\mathcal{O}\rangle_{N-1}\left(\{\lambda\}_{N-1},\left\{z_{\bmod }\right\}_{N-1}\right)$ multiplied by $\sinh (\eta) \prod_{k \neq j} f_{k j} f_{j k}$. But using Eq. (6.15), this demonstrates that the $z$ dependence of the two sides is equal. To investigate the part independent of the $z$ variables, we have to take all of them to zero. In this case, on the rhs only $\mathbb{F}_{N, s}^{\mathcal{O}}\left(\{\lambda\}_{N}\right)$ remains. Since $\langle\mathcal{O}\rangle_{N}\left(\{\lambda\}_{N},\{0\}_{N}\right)=\mathbb{F}_{N, s}^{\mathcal{O}}\left(\{\lambda\}_{N}\right)$, the two sides are equal. This completes the proof.

The previously introduced Eq. [61] is equivalent to Eq. (6.26): One obtains the former equation after dividing by the norm of the Bethe state given by Eq. (5.29).

\section{CONNECTION TO THE THEORY OF FACTORIZED CORRELATION FUNCTIONS}

In the case of the $X X Z$ and $X X X$ spin chains, our results for the current mean values are directly related to certain objects in the theory of factorized correlation functions. In the following, we describe this connection.

Factorization of correlation functions concerns the equilibrium mean values of local operators (for a review, see Ref. [34]). Factorization means that any multipoint correlator can be expressed as sums of products of simple building blocks, which are derived from the two-site density matrix in an inhomogeneous spin chain. On a practical level, the theory consists of two parts: the algebraic part and the physical part. The algebraic part deals with the factorization on the level of the operators, and it is independent of the actual physical situation. On the other hand, the information about the concrete situation is supplied by the physical part of the computation.

The theory was worked out first for the cases of thermal equilibrium in infinite volume and for the ground states in finite volume [79-82]. In Ref. [83], a conjecture was formulated for the physical part in any excited equilibrium state in infinite volume; these formulas have been used to compute the steady-state properties after global quenches. Finally, it was argued in Ref. [84] that the known results for the finite-volume ground states [82] can be extended naturally to all finite-volume excited states, and this leads to the proof of the formulas of Ref. [83] in the thermodynamic limit.

In the following, we summarize the main statements in the case of the $X X X$ chain.

The Bethe vectors are highest weight vectors with respect to the $S U(2)$ symmetry. Let us consider a Bethe state with $\left\langle S_{z}\right\rangle=0$, thus, $N=L / 2$. All such states are $S U(2)$ singlets.

The theory of factorized correlations states $[82,84]$ that in the $S U(2)$ singlet states, any multipoint correlation function can be expressed using only a single generating function $\Psi\left(x_{1}, x_{2}\right)$ that depends on the excited state in question. Defining the coefficients

$$
\Psi_{n, m}=\left.\partial_{x_{1}}^{n} \partial_{x_{2}}^{m} \Psi\left(x_{1}, x_{2}\right)\right|_{x_{1}, x_{2}=0}
$$

it can be shown that all correlators can be expressed as finite combinations of the quantities $\Psi_{n, m}$. The algebraic part of the computation expresses the correlators in terms of $\Psi_{n, m}$, whereas the physical part supplies their actual values, depending on the Bethe state in question.

For example, the simplest $z-z$ correlators can be expressed as

$$
\begin{gathered}
\left\langle\sigma_{1}^{z} \sigma_{2}^{z}\right\rangle=\frac{1}{3}\left(1-\Psi_{0,0}\right), \\
\left\langle\sigma_{1}^{z} \sigma_{3}^{z}\right\rangle=\frac{1}{3}\left(1-4 \Psi_{0,0}+\Psi_{1,1}-\frac{1}{2} \Psi_{2,0}\right), \\
\left\langle\sigma_{1}^{z} \sigma_{4}^{z}\right\rangle=\frac{1}{108}\left[36+288 \Psi_{1,1}-15 \Psi_{2,2}+10 \Psi_{3,1}\right. \\
+\Psi_{2,0}\left(-156+12 \Psi_{1,1}-6 \Psi_{2,0}\right) \\
+2 \Psi_{0,0}\left(-162-42 \Psi_{1,1}+3 \Psi_{2,2}-2 \Psi_{3,1}\right) \\
\left.+\Psi_{1,0}\left(84 \Psi_{1,0}-12 \Psi_{2,1}+4 \Psi_{3,0}\right)\right] .
\end{gathered}
$$

It was shown in Ref. [84] that for the Bethe state $\left|\{\lambda\}_{N}\right\rangle$, the generating function reads

$$
\Psi\left(x_{1}, x_{2}\right)=2 \mathbf{q}\left(x_{1}\right) \cdot G^{-1} \cdot \mathbf{q}\left(x_{2}\right),
$$

where $\mathbf{q}\left(x_{1}\right)$ is a parameter-dependent vector of length $N$ with elements $\mathbf{q}_{j}(x)=q\left(\lambda_{j}-x\right)$ with $q(\lambda)=1 /\left(\lambda^{2}+1 / 4\right)$. Furthermore, $G$ is the Gaudin matrix, which now takes the form

$$
G_{j k}=\delta_{j k}\left(L \frac{1}{u_{j}^{2}+1 / 4}+\sum_{l=1}^{N} \varphi\left(u_{j l}\right)\right)-\varphi\left(u_{j k}\right),
$$

with

$$
\varphi(u)=-\frac{2}{u^{2}+1} .
$$

The factor of 2 is included in Eq. (7.5) to conform with earlier conventions; see Ref. [84].

It follows that the $\Psi_{n, m}$ coefficients can be expressed as

$$
\Psi_{n, m}=2 \mathbf{q}_{n+2} \cdot G^{-1} \cdot \mathbf{q}_{m+2} .
$$

The shifts in the indices are due to our conventions, namely, that the first member of the series of the charges is called $Q_{2}$.

Comparing to Eq. (2.26), we see that the current mean values are

$$
\left\langle\{\lambda\}_{N}\left|J_{\alpha}^{\beta}(x)\right|\{\lambda\}_{N}\right\rangle=\frac{1}{2} \Psi_{\beta-1, \alpha-2} .
$$

This equality gives a new interpretation for the generalized currents: They are special operators that are represented by a single $\Psi_{n, m}$. 
In the case of nonsinglet states, the situation is more involved, and the correlators also involve the so-called moments (see Refs. [84,85]). Nevertheless, the mean values of $S U(2)$-invariant operators are still described by the $\Psi_{n, m}$, and this is in accordance with the fact that in the $X X X$ model, the charges $Q_{\alpha}$ and their currents are also $S U(2)$ invariant.

In the case of the $X X Z$ model, spin-flip-invariant local correlations are described by two generating functions [traditionally denoted as $\omega(x, y)$ and $\omega^{\prime}(x, y)$; see Refs. [79-82]. [It was shown in Ref. [84] that for the finite-volume excited states, the function $\omega(x, y)$ has a form analogous to Eq. (7.5); thus, the Taylor coefficients of this function describe the generalized current operators in the $X X Z$ model.

At present, we do not have an explanation for the observed coincidences between the factorization and the current mean values. Understanding this connection might lead to an independent proof of our results, at least in the $X X Z$ and $X X X$ models.

\section{CONCLUSIONS AND DISCUSSION}

We compute an exact finite-volume formula for the current mean values in Bethe ansatz solvable systems. The main results are Eqs. (2.18) and (2.26). We do not treat the direct thermodynamic limit of these results, but their functional form implies that they reproduce the previously conjectured infinite-volume formulas [see Eq. (2.21) and the discussion there]. We thus supply a rigorous foundation for the treatment of ballistic transport in generalized hydrodynamics.

The task of actually deriving the thermodynamic limit of our formula will be a subject of a separate work. The present result needs to be worked out for the string solutions of the Bethe equations, and it needs to be shown that the semiclassical interpretation carries over to the strings. They describe bound states of spin waves, so they should be treated as separate particles. Afterward, the thermodynamic limit can be taken with standard steps; we will return to this issue in a separate publication.

Perhaps the most interesting finding of this work is that the semiclassical result for the currents remains exact in the interacting quantum theory, even with a finite number of particles. Ultimately, this phenomenon boils down to the two-particle reducibility of the Bethe ansatz wave function [see Eq. (2.8)], which also enables the applicability of the flea gas model to simulate the dynamics [46].

Our rigorous proof is rather general, and it relies on a model-independent form-factor expansion (Theorem 1). However, the expansion itself has to be proven separately, and the techniques to be applied might vary. Here we provide a proof for the case of the Heisenberg spin chains. Whereas we do not treat the Lieb-Liniger model (1D Bose gas) here, the expansion theorem can be worked out with essentially the same techniques (see, e.g., Ref. [63]), at least for those local charges and currents which have welldefined real-space representations [86]. For integrable QFT, the expansion was proven earlier in Ref. [65].

It would be highly desirable to develop an alternative, more transparent proof for the current mean values. For special cases, this can indeed be achieved; for example, the spin current of the $X X Z$ model can be computed using form-factor perturbation theory [87] using similar ideas to those of Ref. [22]. Nevertheless, a simple and general proof is not available. In the special case of the $X X Z$ and $X X X$ models, the connection to the factorized correlation functions might lead to a more transparent derivation.

In this work, we restrict ourselves to local charges on the lattice. However, it is known that there exist quasilocal charges that are essential for the GGE and thus for GHD $[35,36]$. For quasilocal charges, our results hold asymptotically, and the finite-volume formulas (2.18)-(2.26) receive exponentially small corrections. These corrections are due to the long-range contributions to the operators with exponentially decreasing amplitudes.

It would be interesting to extend our results to multicomponent models solvable by the nested Bethe ansatz. In these systems, much less is known about local correlations, and the current operators are good candidates to obtain exact analytic results. In turn, these results will give a rigorous foundation for the hydrodynamic treatment of these models [88]. Also, it would be interesting to consider models without the $U(1)$ symmetry responsible for particle conservation. The integrable $X Y Z$ spin chain is such a model, where the canonical family of conserved charges [74] does not include the $S_{z}$ operator. Nevertheless, there might exist simple exact results for the current operators, and thus also for GHD. We plan to return to these questions in future research.

\section{ACKNOWLEDGMENTS}

We are grateful to Bruno Bertini, Benjamin Doyon, Enej Ilievski, Márton Kormos, Márton Mestyán, Lorenzo Piroli, Gábor Takács, Eric Vernier, Dinh-Long Vu, and Takato Yoshimura for useful discussions. We are thankful in particular to Bruno Bertini and Lorenzo Piroli for drawing our attention to this problem. This research is supported by the BME-Nanotechnology FIKP grant of EMMI (BME FIKP-NAT), by the National Research Development and Innovation Office (NKFIH) (K-2016 Grant No. 119204 and the KH-17 Grant No. 125567), the "Premium" Postdoctoral Program of the Hungarian Academy of Sciences, and Ministry of Human Capacities (EMMI), Hungary.

\section{APPENDIX A: THE ENERGY CURRENT OPERATOR}

Here we investigate a special case of the main formulas (2.18)-(2.26): We consider the energy current $J_{H}$, which is itself a conserved operator in integrable lattice 
models, In fact, it is proportional to the canonical charge $Q_{3}$. For completeness, we rederive this correspondence and show that Eq. (2.18) reproduces the mean values of $Q_{3}$.

The continuity equation for the energy flow is

$$
[H, h(x)]=i\left[J_{H}(x+1)-J_{H}(x)\right],
$$

where $h(x)$ is the Hamiltonian density. It follows that

$$
J_{H}(x+1)=-i[h(x+1), h(x)] .
$$

Using the definition of the boost operator (5.14) and the proportionality relation (5.9), we compute the canonical $Q_{3}$ as

$$
\begin{aligned}
Q_{3}=i\left[B, Q_{2}\right] & =i \kappa^{2}\left[\sum_{x} x h(x), \sum_{y} h(y)\right] \\
& =i \kappa^{2} \sum_{x}[h(x+1), h(x)] .
\end{aligned}
$$

Therefore, we can identify

$$
Q_{3}(x)=-\kappa^{2} J_{H}(x) .
$$

The proportionality factor $-\kappa^{2}$ originates simply in our conventions.

The one-particle eigenvalues of $Q_{3}$ are obtained from the transfer-matrix construction: Eqs. (5.35) and (5.34) give

$$
q_{3}(\lambda)=q_{2}^{\prime}(\lambda)=\kappa e^{\prime}(\lambda) .
$$

For $J_{H}$, Eq. (2.18) takes the form

$$
\left\langle\{\lambda\}_{N}\left|J_{H}(x)\right|\{\lambda\}_{N}\right\rangle=\mathbf{e}^{\prime} \cdot G^{-1} \cdot \mathbf{e} .
$$

We show that after rescaling, this formula gives the expected mean value of $Q_{3}$.

Let us take an $N$-dimensional vector $\mathbf{u}$ whose elements are equal to 1. It follows from the definition of the Gaudin matrix (2.16) that

$$
G \mathbf{u}=L \mathbf{p}^{\prime}
$$

Multiplying by the inverse and using $e(\lambda)=q_{2}(\lambda) / \kappa=$ $-p^{\prime}(\lambda) / \kappa$, we get

$$
\mathbf{e}^{\prime} \cdot G^{-1} \cdot \mathbf{e}=-\frac{1}{\kappa L} \mathbf{e}^{\prime} \cdot \mathbf{u}=-\frac{1}{\kappa^{2} L} \sum_{j=1}^{N} q_{3}\left(\lambda_{j}\right) .
$$

With this results, we indeed obtain

$$
\left\langle\{\lambda\}_{N}\left|Q_{3}(x)\right|\{\lambda\}_{N}\right\rangle=-\kappa^{2}\left\langle\{\lambda\}_{N}\left|J_{H}(x)\right|\{\lambda\}_{N}\right\rangle,
$$

as expected from Eq. (A4).
Equation (A9) is a special case of a more general symmetry relation. It follows from Eq. (5.35) and the symmetry of the Gaudin matrix that the following mean values are equal:

$$
\left\langle\{\lambda\}_{N}\left|J_{\alpha}^{\beta}(x)\right|\{\lambda\}_{N}\right\rangle=\left\langle\{\lambda\}_{N}\left|J_{\beta+1}^{\alpha-1}(x)\right|\{\lambda\}_{N}\right\rangle .
$$

This interesting relation was already observed in GHD in Ref. [12], but a more direct explanation is not yet known.

We stress that the relation above does not mean that the operators $J_{\alpha}^{\beta}(x)$ and $J_{\beta+1}^{\alpha-1}(x)$ are equal; they might differ in total derivatives. The concrete form of the current operators depends on the choice of the charge density representing the global charge operator, and the gauge freedom (5.12) leaves room for redefinitions. It would be interesting to see whether there is a gauge choice which would guarantee that the above relation holds on the level of the operators.

\section{APPENDIX B: EXPLICIT FORMULAS FOR CHARGES AND CURRENTS IN THE $X X X$ CHAIN}

In the $X X X$ Heisenberg chain, the canonical $Q_{2}$ operator (5.7) is

$$
Q_{2}=\frac{1}{2} \sum_{x=1}^{L}\left(\underline{\sigma}_{x} \cdot \underline{\sigma}_{x+1}-1\right) .
$$

Here we use the shorthand notation that $\underline{\sigma}_{x}$ is a vector of operators $\left(\sigma^{x}, \sigma^{y}, \sigma^{z}\right)$ acting on site $x$. Note that $Q_{2}$ is one-half of the Hamiltonian.

Further charges can be computed easily using the boost operator (5.14). After explicit computations, we find

$$
Q_{3}=\sum_{x=1}^{L}\left(-\frac{1}{2}\right)\left(\underline{\sigma}_{x} \times \underline{\sigma}_{x+1}\right) \cdot \underline{\sigma}_{x+2},
$$

$$
\begin{aligned}
Q_{4}= & \sum_{x=1}^{L}\left(\left[\left(\underline{\sigma}_{x} \times \underline{\sigma}_{x+1}\right) \times \underline{\sigma}_{x+2}\right] \cdot \underline{\sigma}_{x+3}-2 \underline{\sigma}_{x} \cdot \underline{\sigma}_{x+1}\right. \\
& \left.+\underline{\sigma}_{x} \cdot \underline{\sigma}_{x+2}+1\right),
\end{aligned}
$$

where the cross denotes the vectorial cross product. Further examples and a closed-form result for all $Q_{\alpha}$ can be found in Ref. [75].

Let us define the generalized current operators $J_{\alpha}^{\beta}$ according to the definition (2.25), with the charge densities as given above. Then, the real-space representations for the first few currents are

$$
\begin{gathered}
J_{2}^{2}(x)=\frac{1}{2}\left(\underline{\sigma}_{x-1} \times \underline{\sigma}_{x}\right) \cdot \underline{\sigma}_{x+1}, \\
J_{3}^{2}(x)=-\left[\left(\underline{\sigma}_{x-1} \times \underline{\sigma}_{x}\right) \times \underline{\sigma}_{x+1}\right] \cdot \underline{\sigma}_{x+2}-\underline{\sigma}_{x} \cdot \underline{\sigma}_{x+1}+1,
\end{gathered}
$$




$$
\begin{aligned}
J_{2}^{3}(x)= & -\frac{1}{2}\left(\left[\left(\underline{\sigma}_{x-2} \times \underline{\sigma}_{x-1}\right) \times \underline{\sigma}_{x}\right] \cdot \underline{\sigma}_{x+1}\right. \\
& \left.+\left[\left(\underline{\sigma}_{x-1} \times \underline{\sigma}_{x}\right) \times \underline{\sigma}_{x+1}\right] \cdot \underline{\sigma}_{x+2}\right) \\
& +\underline{\sigma}_{x} \cdot \underline{\sigma}_{x+1}+\underline{\sigma}_{x-1} \cdot \underline{\sigma}_{x}-\underline{\sigma}_{x-1} \cdot \underline{\sigma}_{x+1}-1 .
\end{aligned}
$$

We can observe the equality $J_{2}^{2}(x)=-Q_{3}(x)$, in accordance with the previous section. Similarly, we find the interesting relation

$$
Q_{4}=-\sum_{x=1}^{L} J_{2}^{3}(x)
$$

which is an analogous identity that follows from Eq. (A10); the minus sign is simply a matter of convention.

\section{APPENDIX C: THE SUMMATION OF THE FORM-FACTOR EXPANSION}

In order to sum up the expansion for the current mean values, we write the rhs of Eq. (4.21) in a slightly different way,

$$
\sum_{\left\{\lambda^{+}\right\} \cup\left\{\lambda^{-}\right\}} \mathcal{J}_{\alpha}\left(\left\{\lambda^{+}\right\}\right) \rho\left(\lambda^{-}\right)=\mathbf{e}^{\prime} \cdot A \cdot \mathbf{q}_{\alpha},
$$

where $\mathcal{J}_{\alpha}(\{\lambda\})$ are the symmetric diagonal form factors of the currents, and we define

$$
A_{j k}=\sum_{\substack{\left\{\lambda^{+}\right\} \cup\left\{\lambda^{-}\right\} \\ \lambda_{j, k} \in\left\{\lambda^{+}\right\}}}\left(\sum_{\mathcal{T}} \prod_{l_{p q} \in \mathcal{T}} \varphi_{p q}\right) \rho\left(\lambda^{-}\right)
$$

Here the summation runs over $\mathcal{T}$, the nondirected spanning trees of $\left\{\lambda^{+}\right\}$. Using this notation, our task is to show that $\sum_{l=1}^{N} G_{j l} A_{l k}=\delta_{j k} \cdot \operatorname{det} G$.

This matrix product can be written out explicitly using the matrix-tree theorem for the Gaudin determinant

$$
\begin{aligned}
& \sum_{l=1}^{N}\left\{\left[\delta_{j l}\left(p_{j}^{\prime} L+\sum_{s=1}^{N} \varphi_{j s}\right)-\varphi_{j l}\right]\right. \\
& \left.\times \sum_{\substack{\left\{\lambda^{+}\right\} \cup\left\{\lambda^{-}\right\} \\
\lambda_{k, l} \in\left\{\lambda^{+}\right\}}}\left(\sum_{\mathcal{T}} \prod_{l_{p q} \in \mathcal{T}} \varphi_{p q}\right)\left(\sum_{\mathcal{F}} \prod_{n \in R(\mathcal{F})} p_{n}^{\prime} L \prod_{l_{u v} \in \mathcal{F}} \varphi_{u v}\right)\right\},
\end{aligned}
$$

where $\mathcal{F}$ denotes the directed spanning forests of $\left\{\lambda^{-}\right\}$. First, we show that the diagonal elements of this matrix product give $\operatorname{det} G$. To do this, we consider the $j=k$ case in Eq. (C3), and we split the outer summation over $l$ into two parts: to the $l=j$ and $l \neq j$ cases.

In the case of $l=j$, the sum of the $\varphi$ terms from the Gaudin matrix appears, multiplying the appropriate terms in $A$. These terms can be written in the following way:

$$
\left(\sum_{\substack{s=1 \\ s \neq j}}^{N} \varphi_{j s}\right) \sum_{\substack{\left\{\lambda^{+}\right\} \cup\left\{\lambda^{-}\right\} \\ \lambda_{j} \in\left\{\lambda^{+}\right\}}}\left(\sum_{\mathcal{T}} \prod_{l_{p q} \in \mathcal{T}} \varphi_{p q}\right)\left(\sum_{\mathcal{F}} \prod_{n \in R(\mathcal{F})} p_{n}^{\prime} L \prod_{l_{u v} \in \mathcal{F}} \varphi_{u v}\right)=\sum_{\substack{s=1 \\ s \neq j}}\left[\varphi_{j s} \sum_{\substack{\left\{\lambda^{+}\right\} \cup\left\{\lambda^{-}\right\} \\ \lambda_{j, s} \in\left\{\lambda^{+}\right\}}}(\ldots)(\ldots)+\varphi_{j s} \sum_{\substack{\left\{\lambda^{+}\right\} \cup\left\{\lambda^{-}\right\} \\ \lambda_{j} \in\left\{\lambda^{+}\right\} \\ \lambda_{s} \in\left\{\lambda^{-}\right\}}}(\ldots)(\ldots)\right] .
$$

Here in the second line, we denote the terms inside the summation with (...) for brevity. Using this equation and renaming the summation variable $l$ to $s$ in Eq. (C3), we finally arrive at the following expression for the diagonal elements of the matrix product $(\mathrm{C} 3)$ :

$$
p_{j}^{\prime} L \sum_{\substack{\left\{\lambda^{+}\right\} \cup\left\{\lambda^{-}\right\} \\ \lambda_{j} \in\left\{\lambda^{+}\right\}}}\left(\sum_{\mathcal{T}} \prod_{l_{p q} \in \mathcal{T}} \varphi_{p q}\right)\left(\sum_{\mathcal{F}} \prod_{n \in R(\mathcal{F})} p_{n}^{\prime} L \prod_{l_{u v} \in \mathcal{F}} \varphi_{u v}\right)+\sum_{\substack{s=1 \\ s \neq j}}^{N}\left[\varphi_{j s} \sum_{\substack{\left.\left.\left\{\lambda^{+}\right\} \cup\left\{\lambda^{-}\right\} \\ j, \in \lambda^{+}\right\} \\ \lambda_{s} \in \lambda^{-}\right\}}}\left(\sum_{\mathcal{T}} \prod_{l_{p q} \in \mathcal{T}} \varphi_{p q}\right)\left(\sum_{\mathcal{F}} \prod_{n \in R(\mathcal{F})} p_{n}^{\prime} L \prod_{l_{u v} \in \mathcal{F}} \varphi_{u v}\right)\right] .
$$

Looking at this expression from the graph theoretical point of view and using the matrix-tree theorem, it can be shown that this sum is indeed $\operatorname{det} G$ : In the first term for every bipartition, the summation over $\mathcal{T}$ (together with the $p_{j}^{\prime} L$ factor) gives the contribution of every such directed spanning tree of $\left\{\lambda^{+}\right\}$in which $\lambda_{j}$ is the root. Together with the summation over $\mathcal{F}$, which is just the contribution from every directed spanning forest of $\left\{\lambda^{-}\right\}$, the first term contains all such directed spanning forests of $\{\lambda\}$ in which $\lambda_{j}$ is one of the roots. On the other hand, in the second term the summation over $\mathcal{T}$ gives the contributions from the free (rootless) spanning trees of $\left\{\lambda^{+}\right\}$. These terms are "connected" to one of the spanning trees in the spanning forests of $\left\{\lambda^{-}\right\}$by the factor $\varphi_{j s}$. Since $\left\{\lambda^{+}\right\}$and $\left\{\lambda^{-}\right\}$are disjoint 
sets, this connection cannot create circles, so the result is still a spanning tree. Furthermore, since we sum over $s$, this connection is realized in every possible way, which means that the second term gives the contribution of every spanning forest of $\{\lambda\}$ in which $\lambda_{j}$ is not among the roots. Altogether, the two terms contain the contribution from all of the directed spanning forests of $\{\lambda\}$, which is just $\operatorname{det} G$ according to the matrix-tree theorem.

For the off-diagonal elements of the matrix product (C3), the same steps can be performed. To show that in this case the result is zero, it is convenient to investigate those terms that contain $p_{j}^{\prime}$, and afterward, those that do not. The ones that contain $p_{j}^{\prime}$ are

$$
\begin{aligned}
p_{j}^{\prime} L & \sum_{\substack{\left\{\lambda^{+}\right\} \cup\left\{\lambda^{-}\right\} \\
\lambda, j, k}}\left(\sum_{\mathcal{T}} \prod_{\left.\lambda^{+}\right\}} \prod_{p q} \in \varphi_{p q}\right)\left(\sum_{\mathcal{F}} \prod_{n \in R(\mathcal{F})} p_{n}^{\prime} L \prod_{l_{u v} \in \mathcal{F}} \varphi_{u v}\right) \\
& -\sum_{\substack{s=1 \\
s \neq j}}^{N}\left[\varphi_{j s} \sum_{\substack{\left\{\lambda^{+}\right\} \cup\left\{\lambda^{-}\right\} \\
\lambda_{k, s} \in\left\{\lambda^{+}\right\} \\
\lambda_{j} \in\left\{\lambda^{-}\right\}}}\left(\sum_{\mathcal{T}} \prod_{l_{p q} \in \mathcal{T}} \varphi_{p q}\right)\right. \\
& \left.\times\left(\sum_{\mathcal{F}} \prod_{\substack{n \in R \mathcal{F} \\
j \in R(\mathcal{F})}} p_{n}^{\prime} L \prod_{l_{u v} \in \mathcal{F}} \varphi_{u v}\right)\right] .
\end{aligned}
$$

In the second line, $j \in R(\mathcal{F})$ denotes that here we consider only those spanning trees of $\left\{\lambda^{-}\right\}$in which $\lambda_{j}$ is one of the roots. Because of this, we can pull out a $p_{j}^{\prime} L$ factor from it. Similar to the previous argument, it can be shown that this whole expression is zero: In the second line, for every partition the spanning tree in $\mathcal{F}$ that originates from $\lambda_{j}$ is connected to a spanning tree in $\mathcal{T}$ by $\varphi_{j s}$, and the result of this connection is still a spanning tree. Since there is a summation over $s$, all possible connections are included. This means that the second line contains all such spanning trees that include $\lambda_{j}$ and $\lambda_{k}$ in addition to all the spanning forests of $\left\{\lambda^{-}\right\}$. But the first line is exactly the same, so their difference is zero. The terms that do not contain $p_{j}^{\prime}$ are

$$
\begin{aligned}
& \sum_{\substack{s=1 \\
s \neq j}}^{N}\left[\varphi_{j s} \sum_{\substack{\left.\left\{\lambda^{+}\right\} \cup\left\{\lambda^{-}\right\} \\
j, k \in \lambda^{+}\right\} \\
\lambda_{s} \in\left\{\lambda^{-}\right\}}}\left(\sum_{\mathcal{T}} \prod_{l_{p q} \in \mathcal{T}} \varphi_{p q}\right)\left(\sum_{\mathcal{F}} \prod_{n \in R(\mathcal{F})} p_{n}^{\prime} L \prod_{l_{u v} \in \mathcal{F}} \varphi_{u v}\right)\right. \\
& -\varphi_{j s} \sum_{\substack{\left\{\lambda^{+}\right\} \cup\left\{\lambda^{-}\right\} \\
\lambda_{k, s} \in\left\{\lambda^{-}\right\} \\
\lambda_{j} \in\left\{\lambda^{-}\right\}}}\left(\sum_{\mathcal{T}} \prod_{l_{p q} \in \mathcal{T}} \varphi_{p q}\right) \\
& \left.\quad \times\left(\sum_{\mathcal{F}} \prod_{\substack{n \in R(\mathcal{F}) \\
j \notin R(\mathcal{F})}}(\mathcal{F}) p_{n}^{\prime} L \prod_{l_{u v} \in \mathcal{F}} \varphi_{u v}\right)\right] .
\end{aligned}
$$

Here in the first line, $\lambda_{j} \in\left\{\lambda^{+}\right\}$and $\lambda_{s} \in\left\{\lambda^{-}\right\}$, and they are connected by $\varphi_{j s}$, while in the second line they are reversed. Since we sum up for every $s$ and every bipartition (and since $\lambda_{j}$ cannot be a root in the second line), the difference is zero. This completes the summation of the expansion.

\section{APPENDIX D: PROOF OF THE SINGULARITY PROPERTIES}

Here we present the proof of the singularity property (6.7), focusing on the case of the $X X Z$ chain. Following the reasoning in Sec. VI, it is sufficient to show that Eq. (6.7) holds for an operator which is the product of the elements of the monodromy matrix:

$$
\mathcal{O}=X\left(\mu_{1}\right), \ldots, X\left(\mu_{M}\right)
$$

where $X(\mu)$ represents one of the four operators: $A, B, C$, $D$. In order to prove Eq. (6.7), we are using the commutation relations of the operators $A, B, C, D$, and the following singularity property of the scalar products (see Sec. IX. 3. of Ref. [23])

$$
\begin{aligned}
\left\langle 0\left|\prod_{j=1}^{N} \mathbb{C}\left(\lambda_{j}^{C}\right) \prod_{j=1}^{N} \mathbb{B}\left(\lambda_{j}^{B}\right)\right| 0\right\rangle \\
\stackrel{\lambda_{N}^{C} \rightarrow \lambda_{N}^{B}}{\longrightarrow} \frac{i \sinh (\eta)}{\lambda_{N}^{C}-\lambda_{N}^{B}}\left(l_{N}^{C}-l_{N}^{B}\right) \prod_{k=1}^{N-1} f_{N k}^{C} f_{N k}^{B}\langle 0| \\
\quad \times \prod_{j=1}^{N-1} \mathbb{C}\left(\lambda_{j}^{C}\right) \prod_{j=1}^{N-1} \mathbb{B}\left(\lambda_{j}^{B}\right)|0\rangle^{\mathrm{mod}} .
\end{aligned}
$$

Here the elements of the sets $\left\{\lambda^{C}\right\}$ and $\left\{\lambda^{B}\right\}$ do not necessarily satisfy the Bethe equations, and the \langle\rangle$^{\bmod }$ notation means that the scalar product is calculated with the modified vacuum expectation values $a^{\text {mod }}$ and $d^{\text {mod }}$ defined in Eq. (6.8).

We stress an important technical detail already at this point: Whereas for the Bethe states we use the renormalized creation operators $\mathbb{B}(\lambda)$ and $\mathbb{C}(\lambda)$ because these are convenient to study the norms and mean values, for the local operator $\mathcal{O}$ we use the original operators $A(\lambda), B(\lambda), C(\lambda)$, $D(\lambda)$ because these are needed for the solution of the inverse problem.

For the sake of completeness, we also present here the commutation relations resulting from the fundamental equation (5.22): 


$$
\begin{aligned}
A(\mu) B(\lambda) & =f(\lambda, \mu) B(\lambda) A(\mu)+g(\mu, \lambda) B(\mu) A(\lambda), \\
B(\mu) A(\lambda) & =f(\lambda, \mu) A(\lambda) B(\mu)+g(\mu, \lambda) A(\mu) B(\lambda), \\
D(\lambda) B(\mu) & =f(\lambda, \mu) B(\mu) D(\lambda)+g(\mu, \lambda) B(\lambda) D(\mu), \\
B(\lambda) D(\mu) & =f(\lambda, \mu) D(\mu) B(\lambda)+g(\mu, \lambda) D(\lambda) B(\mu), \\
A(\lambda) C(\mu) & =f(\lambda, \mu) C(\mu) A(\lambda)+g(\mu, \lambda) C(\lambda) A(\mu), \\
C(\lambda) A(\mu) & =f(\lambda, \mu) A(\mu) C(\lambda)+g(\mu, \lambda) C(\mu) A(\lambda), \\
D(\mu) C(\lambda) & =f(\lambda, \mu) C(\lambda) D(\mu)+g(\mu, \lambda) C(\mu) D(\lambda), \\
C(\mu) D(\lambda) & =f(\lambda, \mu) D(\lambda) C(\mu)+g(\mu, \lambda) D(\mu) C(\lambda), \\
{[A(\lambda), D(\mu)] } & =g(\lambda, \mu)\{C(\mu) B(\lambda)-C(\lambda) B(\mu)\}, \\
{[D(\lambda), A(\mu)] } & =g(\lambda, \mu)\{B(\mu) C(\lambda)-B(\lambda) C(\mu)\}, \\
{[B(\lambda), C(\mu)] } & =g(\lambda, \mu)\{D(\mu) A(\lambda)-D(\lambda) A(\mu)\}, \\
{[C(\lambda), B(\mu)] } & =g(\lambda, \mu)\{A(\mu) D(\lambda)-A(\lambda) D(\mu)\}, \\
{[A(\lambda), A(\mu)] } & =[B(\lambda), B(\mu)]=[C(\lambda), C(\mu)] \\
& =[D(\lambda), D(\mu)]=0,
\end{aligned}
$$

where

$$
f(u, v)=\frac{\sin (u-v+i \eta)}{\sin (u-v)}, \quad g(u, v)=\frac{i \sinh (\eta)}{\sin (u-v)} .
$$

Instead of considering an arbitrary product in Eq. (D1), it is useful to require a specific ordering of the $X=A, B, C$, $D$ operators. It follows from the commutation relations above that any operator of the form $X\left(\mu_{1}\right), \ldots, X\left(\mu_{M}\right)$ can be written as a linear combination of operators in which the order of the $A, B, C$, and $D$ operators is fixed in the following way:

$$
\begin{gathered}
X\left(\mu_{1}\right), \ldots, X\left(\mu_{M}\right)=\sum_{D A A, \ldots, A B B, \ldots, B,} G(\{\mu\}) C C, \ldots, C D D, \ldots,
\end{gathered}
$$

where the $G(\{\mu\})$ are the coefficients of the individual terms, which include combinations of the $f(u)$ and $g(u)$ functions. Because of the linearity of the scalar product, all we need to show is that Eq. (6.7) holds for a single term in this summation.

We first consider products of the form $\mathcal{O}=D\left(\mu_{1}\right)$ $D\left(\mu_{2}\right), \ldots, D\left(\mu_{m}\right) A\left(\mu_{m+1}\right), \ldots, A\left(\mu_{M}\right)$. The possible presence of additional $C$ and $B$ operators is treated later.

First, let us consider only one $A$ operator:

$$
\begin{aligned}
\left\langle 0\left|\prod_{k=1}^{N} \mathbb{C}\left(\lambda_{k}^{C}\right) A(\mu) \prod_{k=1}^{N} \mathbb{B}\left(\lambda_{k}^{B}\right)\right| 0\right\rangle= & a(\mu) \prod_{k=1}^{N} f\left(\lambda_{k}^{B}, \mu\right)\left\langle 0\left|\prod_{k=1}^{N} \mathbb{C}\left(\lambda_{k}^{C}\right) \prod_{k=1}^{N} \mathbb{B}\left(\lambda_{k}^{B}\right)\right| 0\right\rangle \\
& +\sum_{n=1}^{N} a\left(\lambda_{n}^{B}\right) g\left(\mu, \lambda_{n}^{B}\right) \prod_{\substack{k=1 \\
k \neq n}}^{N} f\left(\lambda_{k}^{B}, \lambda_{n}^{B}\right) \frac{d(\mu)}{d\left(\lambda_{n}^{B}\right)}\left\langle 0\left|\prod_{k=1}^{N} \mathbb{C}\left(\lambda_{k}^{C}\right) \mathbb{B}(\mu) \prod_{\substack{k=1 \\
k \neq n}}^{N} \mathbb{B}\left(\lambda_{k}^{B}\right)\right| 0\right\rangle .
\end{aligned}
$$

Here we use only the commutation relation between the operators $A$ and $B$. Taking the $\lambda_{N}^{C} \rightarrow \lambda_{N}^{B}$ limit and using Eq. (D2), we arrive at the expected expression:

$$
\left\langle 0\left|\prod_{k=1}^{N} \mathbb{C}\left(\lambda_{k}^{C}\right) A(\mu) \prod_{k=1}^{N} \mathbb{B}\left(\lambda_{k}^{B}\right)\right| 0\right\rangle \stackrel{\lambda_{N}^{C} \rightarrow \lambda_{N}^{B}}{\longrightarrow} \frac{i \sinh (\eta)}{\lambda_{N}^{C}-\lambda_{N}^{B}}\left(l_{N}^{C}-l_{N}^{B}\right) \prod_{k=1}^{N-1} f_{N k}^{C} f_{N k}^{B}\left\langle 0\left|\prod_{j=1}^{N-1} \mathbb{C}\left(\lambda_{j}^{C}\right) A(\mu) \prod_{j=1}^{N-1} \mathbb{B}\left(\lambda_{j}^{B}\right)\right| 0\right\rangle^{\bmod }
$$

The computation goes similarly for the $D$ operator. Now let us consider the case when $\mathcal{O}=\prod_{k=1}^{M} A\left(\mu_{k}\right)$. To calculate the matrix element of this operator, first we have to compute the effect of it on an arbitrary state:

$$
\prod_{l=1}^{M} A\left(\mu_{l}\right) \prod_{k=1}^{N} \mathbb{B}\left(\lambda_{k}\right)|0\rangle
$$

It is obvious that the result is the linear combination of states with particle number $N$. The rapidities of the particles are coming from the set $\{\mu\}_{M} \cup\{\lambda\}_{N}$. The result can be arranged into a sum, depending on the number of rapidities coming from $\{\mu\}_{M}$. In every term in this summation, we have to take into consideration every possible way that a certain amount of rapidities can be substituted from $\{\mu\}_{M}$. We can thus write the result in the following way,

$$
\prod_{l=1}^{M} A\left(\mu_{l}\right) \prod_{k=1}^{N} \mathbb{B}\left(\lambda_{k}\right)|0\rangle=\sum_{K=0}^{\max (N, M)} \sum_{K} G_{l_{1}, l_{2}, \ldots, l_{K}}^{n_{1}, n_{2}, \ldots, n_{K}}\left(\{\lambda\}_{N} \mid\{\mu\}_{M}\right) \prod_{q=1}^{K} \mathbb{B}\left(\mu_{l q}\right) \prod_{\substack{k=1 \\ k \neq n_{1}, \ldots, n_{K}}}^{N} \mathbb{B}\left(\lambda_{k}\right)|0\rangle,
$$


where $\sum_{K}$ goes over every such subset of $\{\lambda\}_{N} \cup\{\mu\}_{M}$ that has the following two properties: The number of its elements is $N$, and it has exactly $K$ elements coming from $\{\mu\}_{M}$. If $\mu_{l_{1}}, \mu_{l_{2}}, \ldots, \mu_{l_{K}}$ denotes these elements and $\lambda_{n_{1}}, \lambda_{n_{2}}, \ldots, \lambda_{n_{K}}\{\lambda\}_{N}$ those that are not present in the subset, $\sum_{K}$ can be written out explicitly:

$\sum_{K}=\sum_{l_{1}=1}^{M} \sum_{\substack{l_{2}=1 \\ l_{2} \neq l_{1}}}^{M} \ldots \sum_{\substack{l_{K}=1 \\ l_{K} \neq l_{1}, \ldots, l_{K-1}}}^{M} \sum_{n_{1}=1}^{N} \sum_{n_{2}=1}^{N} \ldots \sum_{\substack{n_{K}=1 \\ n_{2} \neq n_{1}}}^{N}$.

The $G$ coefficients can be calculated using only the commutation relations. For example, in the case $K=0$ none of the rapidities are coming from $\{\mu\}_{M}$ and we have

$$
G_{0}^{0}\left(\{\lambda\}_{N} \mid\{\mu\}_{M}\right)=\prod_{l=1}^{M}\left(a\left(\mu_{l}\right) \prod_{k=1}^{N} f\left(\lambda_{k}, \mu_{l}\right)\right) .
$$

Let us calculate now a general coefficient, where $K$ rapidities are coming from $\{\mu\}_{M}$. To do this, we have to keep in mind that every time we exchange the arguments of the operators $A(\mu)$ and $\mathbb{B}(\lambda)$ during commutation, there will appear a factor $g(\mu, \lambda) d(\mu) / d(\lambda)$, while if we do not exchange them, the corresponding factor will be $f(\lambda, \mu)$.

Let $\mu_{l_{1}}$ be the first substituted rapidity and $\lambda_{n_{1}}$ the one which is replaced. In this case, there will be a $g\left(\mu_{l_{1}}, \lambda_{n_{1}}\right) d\left(\mu_{l_{1}}\right) / d\left(\lambda_{n_{1}}\right)$ factor coming from the commutation relation of $A\left(\mu_{l_{1}}\right)$ and $\mathbb{B}\left(\lambda_{n_{1}}\right)$ describing the replacement. The commutation of $A\left(\lambda_{n_{1}}\right)$ and the other $\mathbb{B}$ operators will give the factor $\prod_{\substack{k=n_{1} \\ k \neq n_{1}}} f\left(\lambda_{k}, \lambda_{n_{1}}\right)$. Finally, the effect of $A\left(\lambda_{n_{1}}\right)$ on the pseudovacuum will result in the factor $a\left(\lambda_{n_{1}}\right)$. In the case of the next substituted rapidity $\left(\mu_{l_{2}}\right.$ replacing $\lambda_{n_{2}}$ ), the same factors will appear, expect that now it has to be taken into consideration that one of the commutations will be with $\mathbb{B}\left(\mu_{l_{1}}\right)$ and not with $\mathbb{B}\left(\lambda_{n_{1}}\right)$.

The rest of the substitutions go the same way. The remaining $A$ operators with the nonsubstituted rapidities commute through the $\mathbb{B}$ operators without exchanging the arguments. To take into consideration all possible cases, we have to sum over every $n$ and $l$. But since both the $A$ and $\mathbb{B}$ operators commute with each other, the answer does not depend on which $\lambda$ is replaced by which $\mu$. Therefore, to obtain the right result we have to divide by $K$ !. These steps lead to the following result for a generic coefficient:

$$
\begin{aligned}
G_{l_{1}, l_{2}, \ldots, l_{K}}^{n_{1}, n_{2}, \ldots, n_{K}}\left(\{\lambda\}_{N} \mid\{\mu\}_{M}\right)= & \frac{1}{K !} a\left(\lambda_{n_{1}}\right) g\left(\mu_{l_{1}}, \lambda_{n_{1}}\right) \frac{d\left(\mu_{l_{1}}\right)}{d\left(\lambda_{n_{1}}\right)}\left(\prod_{\substack{k=1 \\
k \neq n_{1}}}^{N} f\left(\lambda_{k}, \lambda_{n_{1}}\right)\right) a\left(\lambda_{n_{2}}\right) g\left(\mu_{l_{2}}, \lambda_{n_{2}}\right) \frac{d\left(\mu_{l_{2}}\right)}{d\left(\lambda_{n_{2}}\right)} \\
& \times\left(\prod_{\substack{k=1 \\
k \neq n_{1}, n_{2}}}^{N} f\left(\lambda_{k}, \lambda_{n_{2}}\right)\right) f\left(\mu_{l_{1}}, \lambda_{n_{2}}\right), \ldots, a\left(\lambda_{n_{K}}\right) g\left(\mu_{l_{K}}, \lambda_{n_{K}}\right) \frac{d\left(\mu_{l_{K}}\right)}{d\left(\lambda_{n_{K}}\right)}\left(\prod_{\substack{k=1 \\
k \neq n_{1}, \ldots, n_{K-1}}}^{N} f\left(\lambda_{k}, \lambda_{n_{K}}\right)\right) \\
& \times\left(\prod_{p}^{K-1} f\left(\mu_{l_{p}}, \lambda_{n_{K}}\right)\right) \prod_{\substack{r=1 \\
r \neq l_{1}, \ldots, l_{K}}}^{M}\left(a\left(\mu_{r}\right) \prod_{\substack{k=1 \\
k \neq n_{1}, \ldots, n_{K}}}^{N} f\left(\lambda_{k}, \mu_{r}\right) \prod_{q \in\left\{l_{1}, \ldots, l_{K}\right\}} f\left(\mu_{q}, \mu_{r}\right)\right) .
\end{aligned}
$$

Multiplying Eq. (D9) from the left with $\langle 0| \prod_{k=1}^{N} \mathbb{C}\left(\lambda_{k}^{C}\right)$ and taking the $\lambda_{N}^{C} \rightarrow \lambda_{N}^{B}$ limit, on the rhs only those terms will give a contribution to the pole, in which $\lambda_{N}^{B}$ is still among the arguments of the $\mathbb{B}$ operators. All these terms will get multiplied by $\left[i \sinh (\eta) / \lambda_{N}^{C}-\lambda_{N}^{B}\right]\left(l_{N}^{C}-l_{N}^{B}\right) \prod_{k=1}^{N-1} f_{N k}^{C} f_{N k}^{B}$ according to Eq. (D2), and the modified scalar products will appear with $N-1$ particles. Since in every such $G\left(\{\lambda\}_{N} \mid\{\mu\}_{M}\right)$ coefficient where $N \notin\left\{n_{1}, \ldots, n_{K}\right\}$ there is an $f\left(\lambda_{N}^{B}, \xi\right)$ factor next to every vacuum expectation value $a(\xi)$, it is true that

$$
\begin{aligned}
G_{l_{1}, l_{2}, \ldots, l_{K}}^{n_{1}, n_{2}, \ldots, n_{K}}\left(\{\lambda\}_{N} \mid\{\mu\}_{M}\right)= & G_{l_{1}, l_{2}, \ldots, l_{K}}^{n_{1}, n_{2}, \ldots, n_{K}}\left(\{\lambda\}_{N-1} \mid\{\mu\}_{M}\right)^{\bmod } \\
& \left(N \notin\left\{n_{1}, \ldots, n_{K}\right\}\right)
\end{aligned}
$$

which means that Eq. (6.7) holds for $\mathcal{O}=\prod_{k=1}^{M} A\left(\mu_{k}\right)$. This computation goes the same way for the product of $D$ operators (only the order of the arguments of the $g$ and $f$ functions are reversed). But because of the linearity of the scalar product, this computation also proves that Eq. (6.7) is true for an operator of the form $\mathcal{O}=$ $D\left(\mu_{1}\right) D\left(\mu_{2}\right), \ldots, D\left(\mu_{m}\right) A\left(\mu_{m+1}\right), \ldots, A\left(\mu_{M}\right)$ : The effect of the products of the $A$ operators on an arbitrary state is a linear combination of states computed above. The effect of the product of the $D$ operators on each term in this linear combination is another linear combination, with coefficients that can be similarly calculated. However, if the original term (the one obtained after acting with the $A$ operators) does not contain $\lambda_{N}^{B}$ among the arguments of the $\mathbb{B}$ operators, then it will not appear there after acting with the $D$ operators. If it is still an argument of a $\mathbb{B}$ operator in the original term, then the appropriate $f$ factor is present next to the VEV, and it will appear also after acting with the $D$ operators. This means that in every term that contains $\mathbb{B}\left(\lambda_{N}^{B}\right)$, the appropriate $f\left(\lambda_{N}, \xi\right)$ or $f\left(\xi, \lambda_{N}\right)$ factor will appear next to the VEV $a(\xi)$ or $d(\xi)$. Therefore, Eq. (6.7) holds for $\mathcal{O}=D\left(\mu_{1}\right) D\left(\mu_{2}\right), \ldots, D\left(\mu_{m}\right) A\left(\mu_{m+1}\right), \ldots, A\left(\mu_{M}\right)$. 
To complete the proof, we consider now the case where there are additional $C$ and $B$ operators. Since they are on the left- and right-hand sides, respectively, they can be considered as additional creation operators in the states. Their presence does not alter the singularity property (6.7) we intend to prove. To see this, let us first consider the most simple case when $\mathcal{O}=$ $C\left(\mu^{C}\right) B\left(\mu^{B}\right)$. The singularities of the form factors of this operator follow from Eq. (D2). First, we consider the renormalized operators:

$$
\begin{aligned}
& \left\langle 0\left|\left(\prod_{j=1}^{N} \mathbb{C}\left(\lambda_{j}^{C}\right)\right) \mathbb{C}\left(\mu^{C}\right) \mathbb{B}\left(\mu^{B}\right)\left(\prod_{j=1}^{N} \mathbb{B}\left(\lambda_{j}^{B}\right)\right)\right| 0\right\rangle \stackrel{\lambda_{N}^{C} \longrightarrow \lambda_{N}^{B}}{\longrightarrow} \frac{i \sinh (\eta)}{\lambda_{N}^{C}-\lambda_{N}^{B}}\left(l_{N}^{C}-l_{N}^{B}\right)\left(\prod_{k=1}^{N-1} f_{N k}^{C} f_{N k}^{B}\right) f\left(\lambda_{N}, \mu^{C}\right) \\
& \quad \times f\left(\lambda_{N}, \mu^{B}\right)\left\langle 0\left|\left(\prod_{j=1}^{N-1} \mathbb{C}\left(\lambda_{j}^{C}\right)\right) \mathbb{C}\left(\mu^{C}\right) \mathbb{B}\left(\mu^{B}\right)\left(\prod_{j=1}^{N-1} \mathbb{B}\left(\lambda_{j}^{B}\right)\right)\right| 0\right\rangle^{\mathrm{mod}} .
\end{aligned}
$$

Substituting the definition (6.1) and also using the modification rule (6.8) for the renormalization on the rhs, we get

$$
\begin{aligned}
& \left\langle 0\left|\left(\prod_{j=1}^{N} \mathbb{C}\left(\lambda_{j}^{C}\right)\right) C\left(\mu^{C}\right) B\left(\mu^{B}\right)\left(\prod_{j=1}^{N} \mathbb{B}\left(\lambda_{j}^{B}\right)\right)\right| 0\right\rangle \stackrel{\lambda_{N}^{C} \rightarrow \lambda_{N}^{B}}{\longrightarrow} \frac{i \sinh (\eta)}{\lambda_{N}^{C}-\lambda_{N}^{B}}\left(l_{N}^{C}-l_{N}^{B}\right)\left(\prod_{k=1}^{N-1} f_{N k}^{C} f_{N k}^{B}\right) \\
& \quad \times\left\langle 0\left|\left(\prod_{j=1}^{N-1} \mathbb{C}\left(\lambda_{j}^{C}\right)\right) C\left(\mu^{C}\right) B\left(\mu^{B}\right)\left(\prod_{j=1}^{N-1} \mathbb{B}\left(\lambda_{j}^{B}\right)\right)\right| 0\right\rangle^{\mathrm{mod}},
\end{aligned}
$$

which has just the desired form. It can be argued similarly, that the presence of the $C$ and $B$ operators does not alter the singularity properties when there are $D$ and $A$ operators present.

[1] H. Spohn, Large Scale Dynamics of Interacting Particles, Texts and Monographs in Physics (Springer-Verlag, Berlin, 1991).

[2] R. Esposito, J. L. Lebowitz, and R. Marra, On the Derivation of Hydrodynamics from the Boltzmann Equation, Phys. Fluids 11, 2354 (1999).

[3] L. P. Pitaevskii and S. Stringar, Bose-Einstein Condensation (Clarendon Press, Oxford, 2003).

[4] D. A. Teaney, in Quark-Gluon Plasma (World Scientific Publishing Co. Pte. Ltd., Singapore, 2010), Vol. 4, pp. 207-266, https://doi.org/10.1142/9789814293297_0004.

[5] R. Grimm, Low-Temperature Physics: A Quantum Revolution, Nature (London) 435, 1035 (2005).

[6] M. Rigol, V. Dunjko, V. Yurovsky, and M. Olshanii, Relaxation in a Completely Integrable Many-Body Quantum System: An Ab Initio Study of the Dynamics of the Highly Excited States of 1D Lattice Hard-Core Bosons, Phys. Rev. Lett. 98, 050405 (2007).

[7] L. Vidmar and M. Rigol, Generalized Gibbs Ensemble in Integrable Lattice Models, J. Stat. Mech. (2017) 064007.

[8] T. Kinoshita, T. Wenger, and D.S. Weiss, A Quantum Newton's Cradle, Nature (London) 440, 900 (2006).

[9] X. Zotos, Ballistic Transport in Classical and Quantum Integrable Systems, J. Low Temp. Phys. 126, 1185 (2002).

[10] O. A. Castro-Alvaredo, B. Doyon, and T. Yoshimura, Emergent Hydrodynamics in Integrable Quantum Systems Out of Equilibrium, Phys. Rev. X 6, 041065 (2016).
[11] B. Bertini, M. Collura, J. De Nardis, and M. Fagotti, Transport in Out-of-Equilibrium XXZ Chains: Exact Profiles of Charges and Currents, Phys. Rev. Lett. 117, 207201 (2016).

[12] B. Doyon and T. Yoshimura, A Note on Generalized Hydrodynamics: Inhomogeneous Fields and Other Concepts, SciPost Phys. 2, 014 (2017).

[13] B. Doyon, Exact Large-Scale Correlations in Integrable Systems Out of Equilibrium, SciPost Phys. 5, 054 (2018).

[14] V. B. Bulchandani, R. Vasseur, C. Karrasch, and J.E. Moore, Bethe-Boltzmann Hydrodynamics and Spin Transport in the XXZ Chain, Phys. Rev. B 97, 045407 (2018).

[15] V. B. Bulchandani, R. Vasseur, C. Karrasch, and J. E. Moore, Solvable Hydrodynamics of Quantum Integrable Systems, Phys. Rev. Lett. 119, 220604 (2017).

[16] B. Doyon and H. Spohn, Drude Weight for the Lieb-Liniger Bose Gas, SciPost Phys. 3, 039 (2017).

[17] E. Ilievski and J. De Nardis, Microscopic Origin of Ideal Conductivity in Integrable Quantum Models, Phys. Rev. Lett. 119, 020602 (2017).

[18] J. De Nardis, D. Bernard, and B. Doyon, Hydrodynamic Diffusion in Integrable Systems, Phys. Rev. Lett. 121, 160603 (2018).

[19] J. D. Nardis, D. Bernard, and B. Doyon, Diffusion in Generalized Hydrodynamics and Quasiparticle Scattering, SciPost Phys. 6, 49 (2019).

[20] M. Schemmer, I. Bouchoule, B. Doyon, and J. Dubail, Generalized Hydrodynamics on an Atom Chip, Phys. Rev. Lett. 122, 090601 (2019).

[21] D.-L. Vu and T. Yoshimura, Equations of State in Generalized Hydrodynamics, SciPost Phys. 6, 23 (2019).

[22] A. Urichuk, Y. Oez, A. Klümper, and J. Sirker, The Spin Drude Weight of the XXZ Chain and Generalized Hydrodynamics, SciPost Phys. 6, 5 (2019). 
[23] V. Korepin, N. Bogoliubov, and A. Izergin, Quantum Inverse Scattering Method and Correlation Functions (Cambridge University Press, Cambridge, England, 1993).

[24] M. Jimbo and T. Miwa, Algebraic Analysis of Solvable Lattice Models, CMBS Regional Conference Series in Mathematics Vol. 85 (American Mathematical Society, Providence, 1995).

[25] N. Kitanine, J. M. Maillet, and V. Terras, Correlation Functions of the XXZ Heisenberg Spin-1/2 Chain in a Magnetic Field, Nucl. Phys. B567, 554 (2000).

[26] F. Göhmann, A. Klümper, and A. Seel, Integral Representations for Correlation Functions of the XXZ Chain at Finite Temperature, J. Phys. A 37, 7625 (2004).

[27] N. Kitanine, J. M. Maillet, N. A. Slavnov, and V. Terras, Master Equation for Spin Spin Correlation Functions of the XXZ Chain, Nucl. Phys. B712, 600 (2005).

[28] N. Kitanine, J. M. Maillet, N. A. Slavnov, and V. Terras, Dynamical Correlation Functions of the XXZ Spin-1/2 Chain, Nucl. Phys. B729, 558 (2005).

[29] H. Boos, M. Jimbo, T. Miwa, F. Smirnov, and Y. Takeyama, Algebraic Representation of Correlation Functions in Integrable Spin Chains, Ann. Inst. Henri Poincaré 7, 1395 (2006).

[30] N. Kitanine, K. K. Kozlowski, J. M. Maillet, N. A. Slavnov, and V. Terras, Algebraic Bethe Ansatz Approach to the Asymptotic Behavior of Correlation Functions, J. Stat. Mech. (2009) P04003.

[31] M. Jimbo, T. Miwa, and F. Smirnov, Hidden Grassmann Structure in the XXZ Model III: Introducing the Matsubara Direction, J. Phys. A 42, 304018 (2009).

[32] N. Kitanine, K. K. Kozlowski, J. M. Maillet, N. A. Slavnov, and V. Terras, A Form Factor to the Asymptotic Behavior of Correlation Functions in Critical Models, J. Stat. Mech. (2011) P12010.

[33] K. K. Kozlowski, Asymptotic Analysis and Quantum Integrable Models, arXiv:1508.06085.

[34] J. Sato, B. Aufgebauer, H. Boos, F. Göhmann, A. Klümper, M. Takahashi, and C. Trippe, Computation of Static HeisenbergChain Correlators: Control over Length and Temperature Dependence, Phys. Rev. Lett. 106, 257201 (2011).

[35] E. Ilievski, M. Medenjak, T. Prosen, and L. Zadnik, Quasilocal Charges in Integrable Lattice Systems, J. Stat. Mech. (2016) 064008.

[36] E. Ilievski, J. De Nardis, B. Wouters, J.-S. Caux, F. H. L. Essler, and T. Prosen, Complete Generalized Gibbs Ensembles in an Interacting Theory, Phys. Rev. Lett. 115, 157201 (2015).

[37] A. C. Cassidy, C. W. Clark, and M. Rigol, Generalized Thermalization in an Integrable Lattice System, Phys. Rev. Lett. 106, 140405 (2011).

[38] H. Bethe, Zur theorie der metalle, Z. Phys. A 71, 205 (1931).

[39] E. Ilievski, E. Quinn, and J.-S. Caux, From Interacting Particles to Equilibrium Statistical Ensembles, Phys. Rev. B 95, 115128 (2017).

[40] B. Pozsgay, The Generalized Gibbs Ensemble for Heisenberg Spin Chains, J. Stat. Mech. (2013) P07003.

[41] M. Fagotti and F. H. L. Essler, Stationary Behaviour of Observables after a Quantum Quench in the Spin-1/2 Heisenberg XXZ Chain, J. Stat. Mech. (2013) P07012.
[42] B. Wouters, J. De Nardis, M. Brockmann, D. Fioretto, M. Rigol, and J.-S. Caux, Quenching the Anisotropic Heisenberg Chain: Exact Solution and Generalized Gibbs Ensemble Predictions, Phys. Rev. Lett. 113, 117202 (2014).

[43] B. Pozsgay, M. Mestyán, M. A. Werner, M. Kormos, G. Zaránd, and G. Takács, Correlations after Quantum Quenches in the XXZ Spin Chain: Failure of the Generalized Gibbs Ensemble, Phys. Rev. Lett. 113, 117203 (2014).

[44] G. Goldstein and N. Andrei, Failure of the GGE Hypothesis for Integrable Models with Bound States, Phys. Rev. A 90, 043625 (2014).

[45] B. Pozsgay, Failure of the Generalized Eigenstate Thermalization Hypothesis in Integrable Models with Multiple Particle Species, J. Stat. Mech. (2014) P09026.

[46] B. Doyon, T. Yoshimura, and J.-S. Caux, Soliton Gases and Generalized Hydrodynamics, Phys. Rev. Lett. 120, 045301 (2018).

[47] M. Gaudin, B. M. McCoy, and T. T. Wu, Normalization Sum for the Bethe's Hypothesis Wave Functions of the Heisenberg-Ising Chain, Phys. Rev. D 23, 417 (1981).

[48] V. E. Korepin, Calculation of Norms of Bethe Wave Functions, Commun. Math. Phys. 86, 391 (1982).

[49] M. Takahashi, Thermodynamics of One-Dimensional Solvable Models (Cambridge University Press, Cambridge, England, 1999).

[50] A. N. Kirillov and V. E. Korepin, Norms of Bound States, J. Sov. Math. 40, 13 (1988).

[51] L. Eisenbud, The Formal Properties of Nuclear Collisions, 1948 (to be published).

[52] E. P. Wigner, Lower Limit for the Energy Derivative of the Scattering Phase Shift, Phys. Rev. 98, 145 (1955).

[53] R. Vlijm, M. Ganahl, D. Fioretto, M. Brockmann, M. Haque, H. G. Evertz, and J.S. Caux, Quasi-Soliton Scattering in Quantum Spin Chains, Phys. Rev. B 92, 214427 (2015).

[54] O. Babelon, D. Bernard, and M. Talon, Introduction to Classical Integrable Systems, Cambridge Monographs on Mathematical Physics (Cambridge University Press, Cambridge, England, 2003).

[55] C. Boldrighini, R. L. Dobrushin, and Y. M. Sukhov, OneDimensional Hard Rod Caricature of Hydrodynamics, J. Stat. Phys. 31, 577 (1983).

[56] G. Mussardo, Off Critical Statistical Models: Factorized Scattering Theories and Bootstrap Program, Phys. Rep. 218, 215 (1992).

[57] M. Mestyán and V. Alba, Molecular Dynamics Simulation of Entanglement Spreading in Generalized Hydrodynamics, arXiv:1905.03206.

[58] F. A. Smirnov, Form-Factors in Completely Integrable Models of Quantum Field Theory, Adv. Ser. Math. Phys. 14, 1 (1992).

[59] B. Pozsgay, W.-V. van Gerven Oei, and M. Kormos, On Form Factors in Nested Bethe Ansatz Systems, J. Phys. A 45, 465007 (2012).

[60] B. Pozsgay and G. Takacs, Form Factors in Finite Volume I: Form Factor Bootstrap and Truncated Conformal Space, Nucl. Phys. B788, 167 (2008).

[61] B. Pozsgay and G. Takacs, Form Factors in Finite Volume II: Disconnected Terms and Finite Temperature Correlators, Nucl. Phys. B788, 209 (2008). 
[62] L. Hollo, Y. Jiang, and A. Petrovskii, Diagonal Form Factors and Heavy-Heavy-Light Three-Point Functions at Weak Coupling, J. High Energy Phys. 9 (2015) 125.

[63] B. Pozsgay, Mean Values of Local Operators in Highly Excited Bethe States, J. Stat. Mech. (2011) P01011.

[64] B. Pozsgay and I. M. Szécsényi, LeClair-Mussardo Series for Two-Point Functions in Integrable QFT, J. High Energy Phys. 5 (2018) 170.

[65] Z. Bajnok and C. Wu, in 2017 MATRIX Annals, edited by J. de Gier, C. E. Praeger, and T. Tao (Springer International Publishing, Cham, 2019), pp. 141-151.

[66] B. Pozsgay, Local Correlations in the 1D Bose Gas from a Scaling Limit of the XXZ Chain, J. Stat. Mech. (2011) P11017.

[67] I. Kostov, D. Serban, and D.-L. Vu, in Quantum Theory and Symmetries with Lie Theory and Its Applications in Physics, edited by V. Dobrev (Springer, Singapore, 2018), Vol. 2, pp. 77-98.

[68] I. Kostov, D. Serban, and D.-L. Vu, Boundary TBA, Trees and Loops, Nucl. Phys. B 949, 114817 (2019).

[69] S. Chaiken and D. Kleitman, Matrix Tree Theorems, J. Comb. Theory Ser. A 24, 377 (1978).

[70] M. Lüscher, Dynamical Charges in the Quantized Renormalized Massive Thirring Model, Nucl. Phys. B117, 475 (1976).

[71] M. G. Tetelman, Lorentz Group for Two-Dimensional Integrable Lattice Systems, Sov. Phys. JETP 1981, 306 (1981), http://www.jetp.ac.ru/cgi-bin/e/index/e/55/2/p306? $\mathrm{a}=$ list.

[72] K. Sogo and M. Wadati, Boost Operator and Its Application to Quantum Gelfand-Levitan Equation for Heisenberg-Ising Chain with Spin One-Half, Prog. Theor. Phys. 69, 431 (1983).

[73] H. B. Thacker, Corner Transfer Matrices and Lorentz Invariance on a Lattice, Physica (Amsterdam) 18D, 348 (1986).

[74] M. Grabowski and P. Mathieu, Structure of the Conservation Laws in Integrable Spin Chains with Short Range Interactions, Ann. Phys. (N.Y.) 243, 299 (1995).
[75] M. P. Grabowski and P. Mathieu, Quantum Integrals of Motion for the Heisenberg Spin Chain, Mod. Phys. Lett. A 09, 2197 (1994).

[76] N. Kitanine, J. M. Maillet, and V. Terras, Form Factors of the $X X Z$ Heisenberg Spin-1/2 Finite Chain, Nucl. Phys. B554, 647 (1999).

[77] F. Göhmann and V.E. Korepin, Solution of the Quantum Inverse Problem, J. Phys. A 33, 1199 (2000).

[78] J. M. Maillet and V. Terras, On the Quantum Inverse Scattering Problem, Nucl. Phys. B575, 627 (2000).

[79] H. Boos and F. Göhmann, On the Physical Part of the Factorized Correlation Functions of the XXZ Chain, J. Phys. A 42, 315001 (2009).

[80] H. E. Boos, J. Damerau, F. Göhmann, A. Klümper, J. Suzuki, and A. Weiße, Short-Distance Thermal Correlations in the XXZ chain, J. Stat. Mech. (2008) P08010.

[81] C. Trippe, F. Göhmann, and A. Klümper, Short-Distance Thermal Correlations in the Massive XXZ Chain, Eur. Phys. J. B 73, 253 (2010).

[82] J. Damerau, F. Göhmann, N. P. Hasenclever, and A. Klümper, Density Matrices for Finite Segments of Heisenberg Chains of Arbitrary Length, J. Phys. A 40, 4439 (2007).

[83] M. Mestyán and B. Pozsgay, Short Distance Correlators in the XXZ Spin Chain for Arbitrary String Distributions, J. Stat. Mech. (2014) P09020.

[84] B. Pozsgay, Excited State Correlations of the Finite Heisenberg Chain, J. Phys. A 50, 074006 (2017).

[85] H. E. Boos, F. Göhmann, A. Klümper, and J. Suzuki, Factorization of Multiple Integrals Representing the Density Matrix of a Finite Segment of the Heisenberg Spin Chain, J. Stat. Mech. (2006) P04001.

[86] B. Davies and V. E. Korepin, Higher Conservation Laws for the Quantum Non-Linear Schroedinger Equation, arXiv: 1109.6604.

[87] We developed a short unpublished proof in collaboration with Lorenzo Piroli.

[88] E. Ilievski and J. De Nardis, Ballistic Transport in the One-Dimensional Hubbard Model: The Hydrodynamic Approach, Phys. Rev. B 96, 081118(R) (2017). 